\title{
Struktur und Mechanismen der Wissenschaft
}

Dieses Kapitel stellt den dritten und letzten Teil des Theorieblocks dar, der als Grundlage für die eigene empirische Analyse dient. Das Kapitel resultiert insofern aus den vorangegangenen Abschnitten, als weder die wissenschaftssoziologischen Theorieansätze zu Transformationen im System der Wissenschaft (siehe Kp. 2) noch die medien- und kommunikations- sowie politikwissenschaftlichen Modelle zu Thematisierungsprozessen (siehe Kp. 3) ein passendes Kategoriengerüst zur Beschreibung und Erklärung von Themenkarrieren in der Wissenschaft bereitstellen. Stattdessen ist also ein eigenes Kategoriengerüst zu entwickeln, das auf die vorliegende Fragestellung zugeschnitten ist. Hierfür werden in diesem Kapitel wissenschaftssoziologische Erkenntnisse zur Funktionsweise der Wissenschaft dargelegt und diskutiert. Sie werden im darauffolgenden Kapitel im Hinblick auf die Fragestellung angepasst, komprimiert und in einen analytischen Rahmen zur Erfassung von Themenkarrieren in der Wissenschaft (siehe Kp. 5) überführt.

Wie funktioniert also die Wissenschaft? Zur Beantwortung dieser Frage ist in erster Linie auf die Erkenntnisse der Wissenschaftssoziologie zurückzugreifen, wobei auch weitere Traditionen, die sich im Feld der Wissenschaftsforschung bewegen, einfließen, insofern sie sich ebenfalls mit den sozialen Mechanismen der wissenschaftlichen Wissensproduktion befassen. Dies gilt insbesondere für die Wissenschaftsphilosophie, Wissenssoziologie, Systemtheorie und die Science and Technology Studies. Es erfolgt ein Zuschnitt dieser Erkenntnisse im Hinblick auf zwei Ziele: (1.) Die theoretischen Grundlagen zur Beantwortung der Forschungsfrage werden geschaffen und eine Einordnung in den bestehenden Wissenskanon ermöglicht und (2.) die Entwicklung einer Forschungsheuristik wird vorbereitet, die den strukturellen Handlungskontext wissenschaftlicher Akteur*innen systematisiert und die empirische Analyse auf die im Sinne der

A. Gravert, Themenkarrieren in der Wissenschaft, Organization \& Public Management, https://doi.org/10.1007/978-3-658-35689-7_4 
Fragestellung relevanten Aspekte fokussiert. Mit Blick auf das empirische Programm dieser Arbeit werden folglich nur solche Erkenntnisse dargelegt, die sich auf die deutschsprachige Planungswissenschaft im Zeitraum 1995-2014 beziehen lassen, die sich also auch mit den in Abschnitt 1.4 aufgeführten Spezifika der heutigen Planungswissenschaft als passfähig erweisen.

Die Erkenntnisse der Wissenschaftssoziologie zu Struktur und Dynamik des sozialen Systems der Wissenschaft werden vor dem Hintergrund der Fragestellung - ,Wie entstehen Themen in der Planungswissenschaft?' - aufgearbeitet. Die Beschaffenheit des institutionellen Rahmens bzw. die personenunabhängigen Regeln und Normen des Wissenschaftssystems werden als Erklärungsmoment für die Entstehung von Themen anvisiert. Im Sinne der Mikrofundierung von Makrophänomenen (siehe Kp. 5) resultieren Themenkarrieren im Verständnis der Arbeit aus den aggregierten (bewussten und unbewussten) Entscheidungen der einzelnen Mitglieder einer Fachgemeinschaft hinsichtlich der Wahl ihrer Forschungsthemen über einen Zeitverlauf. In der Konsequenz lautet die auf die Akteur*innenebene heruntergebrochene Fragestellung: ,Wie wählen wissenschaftliche Akteur*innen ein Thema?`. Im Rückbezug auf das zu erklärende Makrophänomen ergibt sich schließlich die Frage: ,Wie lassen sich Veränderungen der aggregierten Entscheidungen wissenschaftlicher Akteur*innen hinsichtlich ihrer Themenwahl erklären?'.

Dieser Frage folgend verläuft die Darstellung der Struktur und Dynamik der Wissenschaft in drei Abschnitten vom Allgemeinen zum Konkreten. Zunächst werden die Besonderheiten der Wissenschaft sowie deren Einbettung in die gesellschaftliche Umwelt anhand einer differenzierungstheoretischen Sichtweise in den Blick genommen (Kp. 4.1). In dem darauf folgenden Kapitel (4.2) werden Strukturen der inneren Differenzierung der Wissenschaft dargestellt sowie die Mechanismen wissenschaftlicher Erkenntnisproduktion aufgearbeitet. Abschließend werden die drei zentralen Einflussgrößen des institutionellen Kontextes der Wissenschaft - die formale Koordination, die Allokation von Reputation und die Allokation von materiellen Ressourcen - sowie deren Zusammenspiel erläutert (Kp. 4.3).

\subsection{Das System Wissenschaft im Kontext der Gesellschaft}

Um ein grundlegendes Verständnis der Wissenschaft zu erlangen, soll sie zunächst in einer Makroperspektive in den Blick genommen werden. Hierfür wird eine differenzierungstheoretische Sichtweise gewählt, die die Wissenschaft als Teilsystem 
einer funktional differenzierten Gesellschaft beleuchtet. Die Stärken dieser Theorie liegen in der Markierung von Besonderheiten, die das System von anderen sozialen Ordnungen unterscheidet, sowie in der Modellierung von dessen Einbettung in die gesellschaftliche Umwelt anhand struktureller Kopplungen. Aufgrund ihrer Anschlussfähigkeit für institutionalistische Ansätze sowie ihrer Errungenschaften im Feld der Wissenschaftssoziologie sind dabei die Systemtheorie nach Luhmann sowie an sie anschließende Arbeiten (insb. von Schimank, Weingart, Aljets) besonders passfähig für diese Arbeit. Luhmanns Systemtheorie zeichnet sich durch ihre Parallelen mit dem institutionalistischen Programm aus, dem sich auch diese Arbeit zurechnet. Durch die Einbindung von systemtheoretischen Erkenntnissen lassen sich die institutionalistischen Ansätze um ein Verständnis gesellschaftlich bedingter Systemdifferenzen und um die Berücksichtigung struktureller Kopplungen erweitern (Krücken 2002: 15-16; Hasse und Krücken 2015: 94-101). Dies gilt insbesondere für diese Arbeit, da die Systemtheorie wertvolle Erkenntnisse zur Funktionsweise der Wissenschaft und zu ihrer Einbettung in die Gesellschaft bereithält, die bisher in institutionalistischen Ansätzen nicht ausreichend reflektiert sind.

\subsubsection{Der Handlungstypus der Wissenschaft: Die Herstellung von Wahrheit}

Die Systemtheorie nach Luhmann $(1991,1997)$ leistet eine grundlegende Charakterisierung eines Systems, indem sie seine Funktion im Kontext der Gesellschaft sowie einen systemspezifischen Handlungstypus identifiziert, der systeminterne Kommunikationen von anderen Kommunikationen unterscheidet. Wie jedes soziale System nimmt sich also auch die Wissenschaft eines spezifischen gesellschaftlichen Problems an, nämlich dem „Gewinnen neuen, unvertrauten, überraschenden Wissens" (Luhmann 1992: 216). Hieraus ergibt sich gemäß Luhmann die Handlungslogik des Systems: In der Wissenschaft zielen Kommunikationen auf die Verteilung von Wahrheitswerten (wahr und falsch) auf Aussagen ab. Wissenschaftliche Operationen lassen sich auf dieser Grundlage etwa von politischem oder wirtschaftlichem Handeln unterscheiden, weil, so Aljets (2015: 75), „die Frage, ob etwas als,wahr'oder, unwahr'zu behandeln ist, allein nach Maßgabe wissenschaftlicher Kriterien entschieden wird-und nicht etwa unter Gesichtspunkten politischer Macht, massenmedialer Informationswerte oder wirtschaftlicher Zahlungsfähigkeit". Dabei ist die Antwort auf die Frage, was Wahrheit ist, nicht in ,einem besonderen Ding“ zu suchen, so Luhmann 
(1992: 181), sondern sie wird sozial innerhalb des Wissenschaftssystems konstruiert. Dementsprechend muss das Ziel wissenschaftssoziologischer Analysen sein, diese soziale Konstruktion der Wahrheit durch die Wissenschaftler*innen zu beobachten (ebd.).

Die Wahrheitsorientierung wird mit Bezug auf Luhmann häufig als zentrale oder gar einzige Motivation wissenschaftlicher Akteur*innen ausgelegt, ist aber nicht allein tragfähig. Zunächst ist die gemäß Scharpf (1988: 16) „seltsam inkonsistente Zuordnung funktionsspezifischer Codes“ grundsätzlich in Frage zu stellen. So zielt der Code der Wissenschaft (wahr/unwahr) auf „public virtues", während der an anderer Stelle von Luhmann benannte Code des Politiksystems (Macht/keine Macht) „private vices“ repräsentiert. Im Sinne der Konsistenz müsste man aber entweder - analog zum systemspezifischen Streben nach Macht seitens der Politiker*innen - für die Wissenschaftler*innen bspw. das Streben nach Reputation (Reputationserwerb/Reputationsverlust) einsetzen oder umgekehrt - analog zur wissenschaftsspezifischen Orientierung an der Wahrheit - für die Politik eine gesellschaftliche Werteorientierung (bspw. gemeinwohldienlich/gemeinwohlschädlich) vorsehen, , aber nicht hier so und dort anders" (Scharpf 1988: 16-17; Schimank 2010: 233). Scharpf (1988: 17) führt diese Unklarheit auf das ,generelle Desinteresse funktionalistischer Theorien für den prekären Zusammenhang zwischen gesellschaftlicher Funktionserfüllung und den sie stabilisierenden Aktormotiven " zurück. Jedoch äußert sich Luhmann selbst an anderer Stelle zu den Aktormotiven und räumt ein, der Erwerb und die Stabilisierung von Reputation sei ,wesentliches Zweitziel, ja nicht selten auch eingestandenes vorrangiges Ziel wissenschaftlicher Tätigkeit, das man bei der Wahl (oder der Vermeidung) von Themen, Publikationsweisen, Kontakten und Orten des Auftretens nicht ungestraft außer Acht läßt" (Luhmann 1974: 237). Hinzu kämen finanzielle Ressourcen:

„Wenn [...] die logisch technisierte Differenz von wahren und unwahren Aussagen
nicht schon als solche motivierend wirkt, entsteht ein Motivationsdefizit, das besondere
Einrichtungen erforderlich macht, soll eine Bemühung um Erkenntnisgewinn weiterhin
vorkommen. Der moderne Wissenschaftsbetrieb ist hier weithin auf finanzielle Anreize
angewiesen. Er benutzt aber auch einen eigenständig entwickelten Nebencode der
Reputation, in dem Reputationswerte stellvertretend für Wahrheiten zirkulieren und
motivationale Effekte auslösen, die durch Wahrheit selbst nicht mehr zu erwirken sind.“
(Luhmann 2008: 153)

Als zweite Ergänzung ist zu nennen, dass Luhmann neben der reinen Wahrheit zwar auch das „Neue“ und „Überraschende“ des Wissens zum Kriterium für die Bewertung wissenschaftlicher Aussagen erhebt, dies aber nicht in den 
binären Code (wahr/unwahr) integriert (Luhmann 1992: 216-224, s. o.). Vor dem Hintergrund des Prioritäts- und Originalitätsgebots der Wissenschaft erhält die Neuigkeit von Wissen allerdings eine zentrale Bedeutung. Gemäß Weingart (2003: 22-23) ist dieses Prinzip entscheidend für den Reputationserwerb:

„Nur neues Wissen zählt. Wer eine bahnbrechende Entdeckung macht, kann sogar damit rechnen, dass sie mit seinem Namen belegt wird und ihm zu ewigem Ruhm verhilft. Für bereits bekanntes Wissen gibt es in der Wissenschaft dagegen keine Anerkennung. “

Die hohe Bedeutung der Neuigkeit von Wissen lässt sich anhand der gegenwärtigen Veröffentlichungspraxis veranschaulichen, bei der die Überprüfung von Studien und die Replikation von Experimenten in den Hintergrund rücken. Dabei müsste die Erstveröffentlichung eines erfolgreichen Experiments, einer Studie oder einer Theorie bei Zugrundelegung des bloßen Wahrheitscodes ähnliche Aufmerksamkeit hervorrufen und Reputation verschaffen wie die erfolgreiche Bestätigung derselben Ergebnisse. Stattdessen kann nur die Widerlegung bereits veröffentlichter Erkenntnisse erneut Aufmerksamkeit in ähnlichem Maße erzeugen, weil sie neben dem vermeintlichen Wahrheits- auch einen Neuigkeitswert enthält. Eine bloße Fixierung auf den Wahrheitsgehalt als Strukturierungselement und Selektionsprinzip in der Wissenschaft kann also nicht genügen, um die Mechanismen der Wissensproduktion zu erfassen.

Für die weitere Betrachtung der Wissenschaft in dieser Arbeit lässt sich festhalten, dass die soziale Konstruktion wahren und neuen Wissens das zentrale, funktionsspezifische Prinzip des wissenschaftlichen Systems ist. Auf Basis dieses Prinzips bzw. dieser Funktion lässt sich die wissenschaftsspezifische Kommunikationsstruktur erklären: Wahrheit und Originalität sind konditionale Kriterien dafür, dass ein Fachbeitrag langfristig Resonanz in der wissenschaftlichen Gemeinschaft erhalten und dementsprechend Relevanz im Rahmen der wissenschaftlichen Wissensproduktion entfalten kann. In welchem Maße Wissenschaftler*innen Beiträge leisten, die seitens der Fachgemeinschaft für relevant - und folglich auch für wahr und originell - befunden werden, entscheidet wesentlich über die Allokation von Ressourcen, wie bspw. Reputation oder Geldmittel. Gleichzeitig ist es die wissenschaftliche Gemeinschaft selbst, die diese Wahrheit und Neuheit - in der Regel unter Berufung auf für objektiv erachtete Kriterien - diskursiv herstellt. Diese Ausrichtung auf die soziale Konstruktion der Gültigkeit und Neuheit von Fachbeiträgen ist das zentrale Merkmal, das die Wissenschaft von anderen Teilsystemen unterscheidet. Zur Klarstellung sei, auch mit Blick auf die Kritik von Scharpf, 
angemerkt, dass dieses Differenzierungsmerkmal nicht zur Ableitung individueller Handlungsorientierungen im Sinne eines institutionalistischen Verständnisses genügt. Die Identifizierung dieser Handlungsorientierungen erfolgt daher nicht an dieser Stelle, sondern im Zuge der Beschreibung des institutionellen Kontextes der Wissenschaft in Abschnitt 4.3.

\subsubsection{Die Einbettung der Wissenschaft in die Gesellschaft}

Das Wissensschaftssystem ist - trotz seiner oft betonten Autonomie - eingebettet in die Gesellschaft. So wie die Vermittlung wissenschaftlicher Erkenntnisse die gesellschaftliche Umwelt verändert, ist die wissenschaftliche Erkenntnisproduktion - einschließlich der Wahl von Forschungsthemen - durch externe Einflussnahmen beeinflusst. Zur Beleuchtung dieser System-Umwelt-Beziehungen dient in der Systemtheorie der Begriff der strukturellen Kopplung, der die wechselseitige Abhängigkeit und Einflussnahme zwischen System und Umwelt bzw. zwischen zwei Systemen beschreibt (Luhmann 1986: 267; Egner et al. 2008: 15). Aus Sicht der Systemtheorie ist strukturelle Kopplung allerdings nicht als kausale Ursache-Wirkungs-Beziehung zu verstehen, bei der spezifische Handlungen in einem System von außen bestimmt werden können. Stattdessen löst die Systemumwelt Irritationen innerhalb des Systems aus, die eine (nicht-steuerbare) Reaktion des Systems auslösen (Zierhofer 2008: 125). Nicht jedes beliebige Element der Umwelt kann eine Irritation auslösen, sonst würde das System zum Erliegen kommen. Stattdessen bedarf es einer Selektivität bzw. Kanalisierung der Umwelteinflüsse auf irritierbare Bereiche, die mit dem Begriff der strukturellen Kopplung konzeptualisert werden (Luhmann 2005b: 33; Lippuner 2010: 200-201, 2008: 109-110).

Das System Wissenschaft ist auf vielfältige Weise und in zunehmenden Maße mit der gesellschaftlichen Umwelt strukturell gekoppelt (Weingart 2003: 27-30). So werden die für die Wissensproduktion erforderlichen materiellen Ressourcen und weite Teile der organisationalen Strukturen nicht durch die Wissenschaft selbst erzeugt, sondern von anderen gesellschaftlichen Teilsystemen bereitgestellt (Lange und Gläser 2007: 775-776). Zu diesen Leistungen gehören bspw. die Finanzierung von Stellen und technischer Ausstattung, die Vergabe zweckgebundener Forschungsmittel, die Bereitstellung von Publikationsmedien sowie die dazugehörigen Organisationsstrukturen, wie bspw. Universitäten, Forschungsinstitute, Ressortforschungseinrichtungen, Fördermittelgeber*innen, Verlage und 
Bibliotheken. In einer differenzierungstheoretischen Perspektive wird die Wissenschaft daher zwar als autonom in Bezug auf Wahrheitsentscheidungen beschrieben, nicht aber als autark in Bezug auf den Aufbau und Erhalt ihrer Strukturen (Aljets 2015: 76-77).

Diese Abhängigkeit der Wissenschaft von den Leistungen anderer gesellschaftlicher Teilsysteme kann als „Einfallstor“ für verschiedenartige Beeinflussungsversuche durch nicht-wissenschaftliche Akteur*innen gesehen werden. Es ergibt sich ein Spannungsverhältnis zwischen den Autonomiebedürfnissen forschender Akteur*innen auf der einen und den Steuerungsintentionen nicht-wissenschaftlicher Akteur*innen auf der anderen Seite (Aljets 2015: 77). Während das Wissenschaftssystem nach einer möglichst selbstreferenziellen Produktion neuer Erkenntnisse auf der Basis eigener Relevanz-, Originalitäts- und Wahrheitskriterien trachtet, ist die Systemumwelt an spezifischen Leistungen und Ergebnissen des von ihr direkt oder indirekt alimentierten Systems interessiert. Nicht-wissenschaftliche Akteur*innen - etwa aus der Politik, der Wirtschaft oder den Medien - agieren aber auf der Basis jeweils eigener Relevanzkriterien und Nutzenerwartungen und suchen ihre Einflusspotentiale auf die Wissenschaft dahingehend zu nutzen, dass die von ihnen präferierte Forschung begünstigt wird (ebd.).

Der wichtigste Mechanismus zur systemexternen Beeinflussung der Wissensproduktion und der wissenschaftlichen Sozialstruktur erfolgt durch die Finanzierung der Wissenschaft seitens des Staates oder der Wirtschaft. Am stärksten ausgeprägt ist diese strukturelle Kopplung in Formen der Auftragsforschung, in denen wissenschaftsexterne Auftraggeber*innen in unterschiedlichem Maße die Inhalte der Forschung vorzugeben suchen und über die Veröffentlichung der Ergebnisse verfügen möchten. Ebenfalls zu nennen sind Ressortforschungseinrichtungen, die im Grenzbereich zwischen Wissenschaft und Politik organisiert sind und die sowohl an politischen Nutzenerwartungen als auch an wissenschaftsinternen Reputationsmechanismen orientiert sind (Feiertag 2018: 32-34). Auch die finanzielle Unterhaltung der Grundausstattung wissenschaftlicher Einrichtungen ist an bestimmte Erwartungen seitens der anderen Funktionssysteme geknüpft. So finanzieren Unternehmen Forschungseinrichtungen, um sich Innovationen und dadurch Vorteile auf dem Markt zu beschaffen (Lange und Gläser 2007: 776). Der Staat finanziert die Wissenschaft unter anderem als Quelle für Innovationen oder Problemlösungen sowie als Dienstleisterin in der Berufsausbildung. Mit diesen Nutzenerwartungen einher geht eine grundsätzlich präferenzielle Ressourcenzuweisung, die wissenschaftlichen Themen und Disziplinen entgegengebracht (oder vorenthalten) wird. Etwa wurden gemäß Weingart (2003: 29-30) in der Nachkriegszeit die Kernphysik oder in den 1980er und 90er Jahren die 
Molekularbiologie besonders stark gefördert, sodass diesen Disziplinen vorübergehend nicht nur erweiterte Forschungsmöglichkeiten und ein größeres Gewicht auf dem Arbeitsmarkt verliehen wurden, sondern auch ,ein höheres Prestige unter den Disziplinen und die damit einhergehende größere Definitionsmacht". Diese staatliche Einflussnahme auf den Forschungsinhalt wird in der Regel aus der Erwartung einer erhöhten gesellschaftlichen Nützlichkeit hergeleitet, mit der die angestrebte Anpassung (bspw. an Prioritäten des Gesundheitswesens, Cooksey 2006) verbunden ist (Gläser 2012a: 2).

Aus der Fremdfinanzierung der Wissenschaft ergibt sich die Frage, inwiefern wissenschaftsintern verhandelte Forschungsbedarfe und Relevanzkriterien zugunsten wissenschaftsexterner Nutzenerwartungen zurückgestellt oder neu justiert werden. Auch das Interesse der Wissenschaft an einer umgehenden und öffentlichen Kommunikation ihrer Erkenntnisse kann kommerziellen Nutzungs- oder Geheimhaltungsansprüchen der Auftraggeber*innen entgegenstehen. Schließlich ist auch zu fragen, inwieweit universitäre Ausbildungsinhalte allein auf der Basis disziplinärer Wissensbestände und ihrer Reproduktion gestaltet werden bzw. inwieweit auch die systemexterne Nachfrage nach Qualifikationen eine Rolle spielt (vgl. Weingart 2003: 105).

Die Produktion wissenschaftlichen Wissens ist auch durch die Aufmerksamkeitskriterien der Massenmedien ${ }^{1}$ bzw. der durch sie erreichten allgemeinen Öffentlichkeit beeinflusst (Weingart 2003: 116-120). Traditionell werden die Massenmedien als Vermittler zwischen der Wissenschaft und der gesellschaftlichen Umwelt angesehen, die das innerhalb des Wissenschaftssystems produzierte Wissen selektieren, vereinfachen und für die Öffentlichkeit zugänglich machen (Weingart 1998: 869, 2011: 47). Allerdings geht ihre tatsächliche Einflussnahme auf die wissenschaftliche Entwicklung über diese Rollenbeschreibung hinaus. So werden die Massenmedien von Wissenschaftler*innen in Anspruch genommen, wenn im Rahmen von innerwissenschaftlichen Prioritätskonflikten Wissensansprüche schnell und ohne aufwändiges peer review-Verfahren veröffentlicht werden sollen (Weingart 2003: 122). In ihrer Funktion als Beschaffer von Aufmerksamkeit und gesellschaftlicher Legitimation können die Massenmedien die Zuschreibung von Reputation innerhalb der Wissenschaft (positiv wie negativ) beeinflussen (Weingart 2003: 27). Dabei kann Medienprominenz in Konkurrenz zur innerwissenschaftlichen Reputation treten, etwa indem einige

\footnotetext{
${ }^{1}$ Massenmedien werden hier verstanden als „Kommunikationsmittel, die durch technische Vervielfältigung und Verbreitung mittels Schrift, Bild oder Ton Inhalte an eine unbestimmte (weder eindeutig festgelegte noch quantitativ begrenzte) Zahl von Menschen vermitteln und somit öffentlich an ein anonymes, räumlich verstreutes Publikum weitergeben " (Burkart 2002: 169).
} 
Wissenschaftler*innen bewusst öffentliche Bekanntheit suchen, um politisch gesteuerte Ressourcenzuweisungen zu beeinflussen oder um ihre Chancen auf bestimmte Posten in der Wissenschaft zu erhöhen (Goodell 1977). Auch können Wissenschaftler*innen die Entscheidung und Schließung innerwissenschaftlicher Konflikte mit Hilfe gesamtgesellschaftlicher Zustimmung zu ihren Gunsten beeinflussen. Sie wählen diesen „Umweg “ über die Öffentlichkeit mitunter strategisch, wenn etwa ein kontroverses theoretisches Programm etabliert oder eine neue wissenschaftliche Disziplin profiliert werden soll (Weingart 2003: 27-28).

Zwar wird das Erregen von Zustimmung und Prominenz außerhalb der Wissenschaft von den Fachkolleg*innen mitunter geringgeschätzt oder nicht beachtet. Insofern sich gesamtgesellschaftliche Aufmerksamkeit in die innerwissenschaftliche Ressourcenallokation übersetzen lässt, verändert sie aber dennoch Handlungsorientierungen und Strategieoptionen für Wissenschaftler*innen. Hierbei ist auch ein Faktor, dass Universitäten und Forschungsinstitute sowie auch Fördermittelgeber*innen immer stärker ihre mediale Präsenz durch das Vorweisen von Forschungserfolgen zu optimieren suchen, um sich in der Konkurrenz mit anderen Einrichtungen zu profilieren (Weingart 2015: 113-114, 2017). Auch richten hochrangige Fachzeitschriften wie bspw. Nature und Science ihre Redaktionspolitik nicht allein an wissenschaftlichen Kriterien aus, sondern auch an medialem Nachrichtenwert. Dieses Selektionsprinzip hat wiederum Rückwirkungen auf die Verteilung von Ressourcen sowie auf die Handlungsbedingungen von Wissenschaftler*innen, da eine Platzierung von Forschungsergebnissen in diesen Zeitschriften von Fördermittelgeber*innen und Wissenschaftler*innen angestrebt wird (Franzen 2012).

Die gesellschaftliche Öffentlichkeit nimmt darüber hinaus zunehmend aktiv Einfluss auf die wissenschaftliche Entwicklung, etwa indem Bürger*innen im Rahmen von citizen science Wissen produzieren, oft in Zusammenarbeit mit oder unter Aufsicht von professionellen Wissenschaftler*innen. Insbesondere in der Planungswissenschaft ist zu berücksichtigen, dass gesellschaftliche Bewegungen und Bürger*inneninitiativen (bspw. „Recht auf Stadt") sich auf vielfältige Weise in der Wissenschaft niederschlagen und Übergänge oft fließend sind. Auch das crowd funding wissenschaftlicher Forschung ist zu nennen, bei dem die Öffentlichkeit, unabhängig von innerwissenschaftlichen Relevanzkriterien, über die Finanzierung und damit die Durchführbarkeit bestimmter Projekte (mit-) bestimmt.

Ein anders gelagertes Beispiel für die gesellschaftliche Beeinflussung ist die partielle Übernahme gesellschaftlicher Vorbedingungen und Wertestrukturen in das Wissenschaftssystem. Hierbei sind nicht nur die sich verändernden normativen Bewertungskriterien bei der Wahl von Methoden und Gegenständen, 
insbesondere was den Ausschluss bestimmter Forschungen betrifft (bspw. Tierversuche), zu nennen. Auch gesellschaftliche Diskriminierungen, etwa von Frauen oder ethnischen Minderheiten, schlagen sich in der Wissenschaft nieder (Weingart 2003: 28-29). So verfügen Teile der Gesellschaft (bspw. Wissenschaftler*innen aus den Entwicklungsländern) bereits über defizitäre Eintrittsbedingungen in die Wissenschaft. Ebenso tragen sich strukturelle gesellschaftliche Benachteiligungen (bspw. gegenüber Frauen) in Benachteiligungen innerhalb der Wissenschaft fort. In der Folge liegt etwa der Frauenanteil unter den höchstdotierten C4/W3Professuren bei lediglich 11,6 \% (DFG 2019a). Zwar ist die wissenschaftliche Gemeinschaft sicher nicht als Spiegel der Gesellschaft zu betrachten, in Bezug auf bestimmte Fragen ist aber zu vermuten, dass strukturell dominierende Sichtweisen sowie Priorisierungen von Themen und Meinungen in der wissenschaftlichen Gemeinschaft in ähnlichen, sich verändernden, Ausprägungen vorliegen, wie im öffentlichen oder politischen Diskurs.

Zusammenfassend ist zu konstatieren, dass die Wissenschaft von der Bereitstellung finanzieller Ressourcen und organisationaler Strukturen abhängt und dass diese Leistungen mit spezifischen - auch inhaltlichen - Erwartungen verknüpft sind. Die Wissenschaft ist zudem von außerwissenschaftlichen Relevanzkriterien und gesellschaftlichen Bedingungen beeinflusst. Aus den jeweils unterschiedlichen Handlungslogiken ergibt sich, dass sich Erwartungen und Relevanzkriterien von System zu System unterscheiden. Ein Forschungsergebnis kann dementsprechend, je nach Kontext, ganz unterschiedliche Bedeutungen und Folgen haben, so Aljets (2015: 79):

\begin{abstract}
„Im Wirtschaftssystem kann das Forschungsergebnis als Chance für ein neues Geschäftsmodell oder als die Bedrohung eines etablierten Vertriebswegs eingeschätzt werden. Im politischen System können daraus Gefahren des Machterhalts oder Potentiale der Machtgewinne resultieren, weil sich das Forschungsergebnis für oder gegen eine etablierte politische Position einsetzen lässt. In den Massenmedien wiederum kann die Erkenntnis der Forschung einen eigenständigen Informationswert haben, der sich aus den zentralen Nachrichtenwerten des massenmedialen Systems [ergibt], statt aus den wissenschaftlichen Kriterien des Forschungssystems. "
\end{abstract}

Da sich die verschiedenen Funktionssysteme der Gesellschaft ständig wandeln, verändern sich auch die von außen an die Wissenschaft herangetragenen Prioritäten und Beurteilungskriterien. Durch verschiedene Kanäle vermittelt, ergibt sich ein Interdependenzverhältnis, das wesentlich zum institutionellen Kontext der Wissenschaft beiträgt. In dem Maße wie die Wissenschaft auf die sich verändernden externen Erwartungen reagiert, wird die gesellschaftliche Umwelt zur 
treibenden Kraft für den innerwissenschaftlichen Wandel (Schimank 2006; Aljets 2015: 77).

Im Sinne des Konzepts der strukturellen Kopplung erfolgt dieser Wandel allerdings nicht im direkten Durchgriff von außen. Stattdessen verändert die gesellschaftliche Umwelt bestimmte Rahmenbedingungen, auf Grundlage derer das selbstreferenziell geschlossene wissenschaftliche Kommunikationssystem eine Reaktion entwickelt, die nur bedingt steuer- und vorhersehbar ist. Die Beeinflussungsversuche der Umwelt konstituieren also, vermittelt über strukturelle Kopplungen, einen Teil des Handlungskontextes, auf Grundlage dessen wissenschaftliche Akteur*innen eigenständig handeln. Letztlich entscheidet aber die Wissenschaft selbst darüber, ,welche Fragen es wert sind, durch Forschung beantwortet zu werden, und welche nicht" (Weingart 2003: 33-34). Für die Untersuchung der Frage, wie die Wissenschaft zu diesen Entscheidungen gelangt, ist von der Ebene des gesellschaftlichen Teilsystems auf die Ebene der wissenschaftlichen Gemeinschaft zu wechseln.

\subsection{Die Fachgemeinschaft als Ort wissenschaftlicher Erkenntnisproduktion}

In diesem Teilkapitel wird die Makroebene verlassen und eine Mesoperspektive eingenommen, die die Fachgemeinschaft als Ort der wissenschaftlichen Erkenntnisproduktion fokussiert. Ein wesentliches Ziel dieser Betrachtung ist es, basale Kategorien (bspw. Fachgemeinschaft, Zitation) und Mechanismen (bspw. Publizieren zur Erlangung von Reputation) darzulegen, die für die Ableitung eines eigenen analytischen Rahmens benötigt werden. Hierfür kommen Modelle und Theorien zur Anwendung, die sich mit der Koordination der Wissenschaft bzw. in einem breiteren, soziologischen Verständnis mit der Governance der Wissenschaft befassen. Dieser Perspektive lassen sich unterschiedliche Ansätze zuordnen, wie insbesondere Science Policy Studies, organisationssoziologische oder akteurtheoretische Ansätze.

Im Vergleich mit anderen gesellschaftlichen Teilsystemen wird der Wissenschaft ein hoher Grad an Autonomie zugesprochen. Die Grundlage für diese Spezifität ist, dass die wissenschaftliche Gemeinschaft - zumindest in der Idealvorstellung - über ein ,von allen anderen sozialen Systemen abgegrenztes Aufmerksamkeitsregime“ verfügt (Weingart 2015: 111). Schließlich sind wissenschaftliche Akteur*innen nicht wie bspw. die Massenmedien an einer systemexternen Kundschaft orientiert, sondern sie sind ihr eigenes Publikum 
(Luhmann 1992: 625; Debackere et al. 1994: 26-27). So konstatiert Bourdieu (1975: 23):

„[...] [Scientific] producers tend to have no possible clients other than their competitors (and the greater the autonomy of the field, the more this is so). This means that in a highly autonomous scientific field, a particular producer cannot expect recognition of the value of his products (, reputation', ,prestige', ,authority', ,competence', etc.) from anyone except other producers, who, being his competitors too, are those least inclined to grant recognition without discussion and scrutiny. “

Die primären Konsument*innen neuen Wissens sind also gleichzeitig dessen Produzent*innen und stehen somit in Konkurrenz zueinander. Letztlich können nur die Fachkolleg*innen die Qualität wissenschaftlicher Erzeugnisse bewerten und auf dieser Basis die Urheber*innen belohnen oder sanktionieren. Eine Konsequenz aus dieser Besonderheit ist, dass die Fachgemeinschaft der Verfolgung eigener und selbst definierter Zielsetzungen verhältnismäßig hohe Priorität beimessen kann (Hasse 2012: 52). Das peer review-System sowie die akademische Selbstverwaltung sind Beispiele für die Institutionalisierung dieses Prinzips (Heidler 2010: 14-15).

\subsubsection{Binnendifferenzierung der Wissenschaft: Disziplin und Fachgemeinschaft}

Das abgeschlossene Aufmerksamkeitsregime der Wissenschaft schlägt sich in einem ausdifferenzierten internen Kommunikationssystem nieder. Horizontal ist die Wissenschaft in mehr oder weniger formalisierte soziale Einheiten gegliedert, die sich um jeweils spezifische Forschungsthemen, Methoden und Theorien gruppieren. Diese Binnendifferenzierung der Wissenschaft ist zum einen die Beschreibung eines Zustands, sie ist aber auch ein fortlaufender Prozess. So können sich Grenzen von Disziplinen, Fakultäten oder Fachbereichen ausweiten oder kontrahieren. Die zwei Triebkräfte dieser Binnendifferenzierung sind zum einen das stetige Wachstum der Wissenschaft als Ganzes, also insbesondere die zunehmende Anzahl wissenschaftlicher Akteur*innen. Zum anderen fächern sich die Disziplinen aufgrund der zunehmenden wissenschaftlichen Abstraktion und der selbstreferenziellen Konstituierung neuer Gegenstände immer weiter auf (Weingart 2003: 37-38). Der zunehmende Apell nach trans- und interdisziplinärer Forschung konterkariert einerseits die fortlaufende Ausdifferenzierung der Wissenschaft, ist aber auf der anderen Seite auch Ausdruck ebendieser Entwicklung (ebd.: 53). 
Es gibt unterschiedliche Ansätze die Einheiten der Wissensproduktion zu benennen (Disziplinen, Fakultäten, Forschungs- und Arbeitsbereiche, Fachgemeinschaften usw.), zu definieren, voneinander abzugrenzen und zu hierarchisieren. Als basale Differenzierungskategorie dient die Untergliederung in Disziplinen. Diese haben sich historisch primär aufgrund eines über längere Zeit konstanten thematischen Alleinstellungsmerkmals sowie einer gewissen Größe formell institutionalisiert. Disziplinen sind jeweils an verschiedenen Universitäten und Forschungsinstituten organisiert und strukturieren die Rahmenbedingungen der Forschung. Unter anderem ist die soziale Organisation der Wissensproduktion einer Disziplin durch ihr Maß an „attention space“ (Collins 1998) repräsentiert und beeinflusst, also der Anzahl wissenschaftlicher Beiträge, die in Zeitschriften, auf Konferenzen und in anderen Formaten untergebracht und rezipiert werden (Hackett et al. 2017: 741). In der deutschsprachigen Wissenschaftssoziologie verbreitet ist die Definition von Disziplinen nach Stichweh (2014: 17):

\begin{abstract}
„Disziplinen sind Formen sozialer Institutionalisierung eines mit vergleichsweise unklareren Grenzziehungen vorlaufenden Prozesses kognitiver Differenzierung der Wissenschaft. Zur Identifizierung und Charakterisierung einer ,Disziplin 'verweisen wir typischerweise: 1) auf einen hinreichend homogenen Kommunikationszusammenhang von Forschern - eine , scientific community“; 2) auf einen Korpus wissenschaftlichen Wissens, der in Lehrbüchern repräsentiert ist, d.h. sich durch Kodifikation, konsentierte Akzeptation und prinzipielle Lehrbarkeit auszeichnet; 3) eine Mehrzahl je gegenwärtig problematischer Fragestellungen; 4) einen , set ‘von Forschungsmethoden und paradigmatischen Problemlösungen; 5) eine disziplinenspezifische Karrierestruktur und institutionalisierte Sozialisationsprozesse, die der Selektion und ,Indoktrination' des Nachwuchses dienen. “
\end{abstract}

Fach- oder Arbeitsbereiche lassen sich als weitere Binnendifferenzierung von Disziplinen anhand der genannten Kriterien - mit Ausnahme der bereichsspezifischen Karrierestruktur - verstehen. Zwischen einzelnen Disziplinen und Fachbereichen bestehen jeweils Überlappungen (bspw. in Bezug auf die Forschungsfragen oder die Mitglieder) und es lassen sich weitere Binnendifferenzierungen bzw. Subsumierungen vornehmen (bspw. Stadtgeografie als Teilmenge der Humangeografie) (vgl. Laudel und Gläser 2007: 103-104).

Innerhalb von Disziplinen und über Disziplinen hinweg lassen sich darüber hinaus Fachgemeinschaften abgrenzen, also informelle Kommunikationszusammenhänge von Akteur*innen, in denen kollektiv ein jeweils spezifisches Wissen produziert wird (Gläser 2012b). In Abgrenzung zu Disziplinen und Fachbereichen 
sind Fachgemeinschaften unabhängig vom Grad ihrer (formellen) Institutionalisierung zu denken, was nicht ausschließt, dass sie in formalisierten Organisationszusammenhängen repräsentiert sind. Die Mitglieder einer Fachgemeinschaft sind potenziell in unterschiedlichen Disziplinen verortet, arbeiten aber an einem zusammenhängenden Problem-Set. Anhand des Problem-Sets lassen sich gemäß Debackere et al. (1994: 22-23) drei Typen von Fachgemeinschaften abgrenzen: Sie beschäftigen sich entweder mit demselben Thema (bspw. Schrumpfende Städte), nutzen dieselben Techniken und Methoden (bspw. Netzwerkforschung) oder verfolgen das gleiche theoretische Programm (bspw. Neo-Institutionalismus) (ebd.).

Die Mitglieder einer Fachgemeinschaft kommunizieren ihre Forschungsergebnisse einander und verwenden diese im Rahmen ihrer jeweils eigenen Forschung, was sich wiederum im Zitat dokumentiert (Weingart 2003: 32-33). Sie heben sich somit als hinreichend verdichtete Kommunikationsgemeinschaften von Forscher*innen aus der wissenschaftlichen Gemeinschaft hervor und lassen sich anhand von Ko-Autorenschafts- oder Zitationsbeziehungen identifizieren (vgl. Bettencourt et al. 2009: 210; siehe Kp. 2.3). Die Entstehung einer Fachgemeinschaft hat verschiedene Ursachen, wie Hackett et al. (2017: 739) auf Basis einer Metaanalyse aufzählt: Neue Techniken und Methoden lassen neue Fragestellungen in den Blick rücken, für (neues) wissenschaftliches Wissen werden neue Verwendungsmöglichkeiten erkannt, eine wachsende Gruppe erkennt eine Frage oder ein Problem als vielversprechend an, Forschungsgegenstände, die mehrere etablierte Fachgebiete vereinen, werden verstärkt beleuchtet, einflussreiche Interessengruppen werden auf einen Gegenstand aufmerksam.

Weil die formell institutionalisierten Strukturen der Wissenschaft in Form von Disziplinen, Fakultäten oder Fachbereichen die tatsächliche ,tribale Organisation wissenschaftlichen Wissens oft nur sehr grob, unvollständig und mit einer Verzögerung von Jahrzehnten " (Angermüller 2012: 712) abbilden, erhalten die informell emergierenden Fachgemeinschaften - bzw. scientificlepistemic/imagined communities - als eigentliche Orte der Wissensproduktion unabhängig vom Grad ihrer Institutionalisierung traditionell besonderes Augenmerk in der Wissenschaftssoziologie (Gläser et al. 2017: 984). Sie werden als ,genuine soziale Form wissenschaftlicher Wissensproduktion“ (Braun-Thürmann 2010: 73), ,kollektive Wissensproduzenten“ (Gläser 2012a: 7) und als die „entscheidenden Wissensorte“ (Glückler und Goeke 2009: 266) angesehen: „Vor allem hier sollte Wissen geschaffen, widerlegt und somit fortwährend verändert werden " (ebd.). Ihr geteiltes Wissen sowie ihre Präferenzen und Normen sind maßgeblich für die Ableitung neuer Forschungsprobleme und Lösungsansätze durch ihre Mitglieder, wie Gläser (2012a: 7) ausführt: 
„The preferences and norms of the scientific community provide important information about acceptable contributions and contributions that would earn a researcher the highest reputation. Scientific communities tacitly or explicitly agree on the problems that must be urgently solved because the community's further progress depends on the solution."

Die fortlaufende Produktion neuer Erkenntnisse ist Bedingung für den Fortbestand der Fachgemeinschaft, allerdings ist die damit verbundene Modifizierung oder Revidierung etablierter Wissensbestände gleichzeitig auch die Grundlage für das Aussterben, die Neuentstehung oder die Metamorphose von Fachgemeinschaften (Aljets 2015: 75).

Die Mitgliedschaft zu einer Fachgemeinschaft wird nicht durch die Entscheidung einer zentralen Instanz erworben, sondern dadurch, dass ein*e Wissenschaftler*in einen eigenen wissenschaftlichen Beitrag zum geteilten Wissen hinzufügt und dieser Beitrag von den anderen Mitgliedern der Gemeinschaft rezipiert wird (Aljets 2015: 76). Um überhaupt einen Beitrag einspeisen zu können, gelten jedoch institutionalisierte Teilnahmebedingungen (vgl. Weingart 2003: 32-33). So wird häufig die Zertifizierung wissenschaftlicher Kompetenz nach Abschluss eines Ausbildungsgangs, also ein akademischer Grad, sowie die Anstellung an einer Universität oder an einem Forschungsinstitut explizit oder implizit zur Bedingung erhoben, um einen Beitrag in einer Fachzeitschrift oder auf einer wissenschaftlichen Konferenz veröffentlichen zu können. Ein weiterer Selektionsmechanismus ist das peer review-Verfahren, also die formalisierte Überprüfung und Beurteilung von Publikationen und Forschungsanträgen durch Mitglieder der Fachgemeinschaft auf der Grundlage fachgemeinschaftlicher Qualitätskriterien.

Die Qualität und Quantität der fachgemeinschaftlichen Resonanz auf die wissenschaftlichen Beiträge ihrer Mitglieder bilden die wesentliche Grundlage für die Vergabe von Reputation, materiellen Ressourcen und Positionen. Entlang dieser Faktoren differenziert sich die Sozialstruktur der Fachgemeinschaft vertikal aus. Daran anknüpfend stellt Aljets (2015: 75) fest:

\footnotetext{
„Zusammengenommen ergibt sich eine Struktur des Wissenschaftssystems, bei der sich die horizontal differenzierten Fachgemeinschaften vertikal entlang der erworbenen Reputation (und organisationalen Positionen) differenzieren. Die Grenzen innerhalb der Struktur ergeben sich daraus, dass die Reputation, die durch eine Fachgemeinschaft zugeschrieben wird, nicht ohne weiteres in eine andere übertragen werden kann."
} 


\title{
4.2.2 Soziale Mechanismen der fachgemeinschaftlichen Wissensproduktion
}

Nachdem im vorangegangenen Kapitel die Fachgemeinschaften als die entscheidenden kollektiven Wissensproduzentinnen beschrieben wurden, soll nun fokussiert werden auf die konkreten, sozialen Mechanismen dieser Wissensproduktion. Dabei wird nicht an der auf individueller Ebene anzusiedelnden Forschung - empirische Analyse, Datenauswertung, kognitive Entwicklung usw. - angesetzt. Stattdessen werden die auf fachgemeinschaftlicher Ebene ablaufenden kollektiven Prozesse - Formulierung der Erkenntnis, Publikation, peer review, Rezeption, Zitation, Allokation von Reputation, materiellen Ressourcen und Positionen - beschrieben.

Der hier zu schildernde idealtypische Mechanismus fachgemeinschaftlicher Erkenntnisproduktion beginnt mit der Formulierung wissenschaftlicher Erkenntnisse durch Forscher*innen. Sie speisen diese Erkenntnisse anhand von Fachbeiträgen - Publikationen, Vorträgen, Kommentaren usw. - in die fachöffentliche Kommunikation ein. Das maßgebliche Medium für einen Fachbeitrag ist die wissenschaftliche Publikation, also die Veröffentlichung wissenschaftlicher Ergebnisse in einer Monografie, als Beitrag in einem Sammelband oder als Aufsatz in einer wissenschaftlichen Fachzeitschrift. In referierten Zeitschriften und Sammelbänden werden Fachbeiträge vor ihrer Veröffentlichung, wie auch Anträge zur Einwerbung von Forschungsmitteln, in einem mehr oder weniger formalisierten Verfahren einem peer review unterzogen. Dabei handelt es sich um eine erste Prüfung durch in der Regel nicht mehr als zwei unabhängige Gutachter*innen, mit dem Ziel die Signifikanz, Aktualität, Originalität, Validität und Plausibilität des Beitrags zu gewährleisten. Weingart (2003: 25) sieht in dieser fachgemeinschaftlichen Begutachtung den

\begin{abstract}
„[...] zentrale[n] Entscheidungsmechanismus in der Wissenschaft, über den sowohl das Erscheinen neuen Wissens als auch die Verteilung von Ressourcen zur Produktion zukünftigen Wissens entschieden wird. Die Gefahr, dass sich verdiente Reputation in illegitime Macht wandelt und die Funktion des gatekeepers missbraucht wird, erscheint als das größte Risiko der Wissenschaft. Es ist aufgrund dieser strategischen Stellung der peer review also kaum überraschend, dass Infragestellungen und/oder Funktionsstörungen außerordentlich sensible Reaktionen zur Folge haben. “
\end{abstract}

Nach der Veröffentlichung des Wissens können die Fachkolleg*innen den Beitrag rezipieren, bewerten und im Rahmen der eigenen Forschung weiterverwenden. Die wissenschaftliche Relevanz des Beitrags äußert sich darin, ob und wie das 
produzierte Wissen in der nachfolgenden Wissensproduktion der wissenschaftlichen Gemeinschaft Verwendung findet und zitiert wird (Morris und Van der Veer Martens 2008: 219-220). Sie entscheidet sich also erst durch die Quantität (,wieviele beziehen sich auf den Beitrag?‘) und die Qualität (bspw. ,wer bezieht sich wie auf den Beitrag?') der Reaktionen der Fachgemeinschaft nach der Veröffentlichung.

In wissenschaftlichen Publikationen rekurrieren die Autor*innen auf die Werke anderer Wissenschaftler*innen und dokumentieren dies - so zumindest das Ideal wissenschaftlicher Redlichkeit - durch Zitationen. Dabei lassen sich zwei Funktionen der Zitation unterscheiden: die formale Funktion (Offenlegung der Quellen) und die latente Funktion (Verteilung und Entzug von sozialer Anerkennung bzw. Reputation). Aus der formalen Funktion ergibt sich das Verständnis einer Zitation als sichtbares Element eines diachronen Wissensnetzwerks. Demnach indizieren Zitationen, so Aufenvenne und Steinbrink (2014: 263-264):

„[...] eine inhaltliche Bezugnahme und somit eine Wissensbeziehung zwischen dem
zitierenden und dem zitierten Autor. Das Schreiben wissenschaftlicher Texte lässt sich
sodann als ein interaktiver Netzwerkprozess verstehen, in dem Zitationen als textliche
Spur einer Wissensbeziehung begreifbar und als Teil eines Wissensnetzwerks analytisch
erfassbar werden.“

Zitationen gehören zu den wesentlichen Faktoren und Indikatoren für die Allokation von sozialer Anerkennung bzw. wissenschaftlicher Reputation. In dieser durch das Zitieren und Nicht-Zitieren vermittelten Belohnung und Sanktionierung ist die latente Funktion der Zitation angelegt. In Literaturverzeichnissen manifestieren sich folglich nicht nur Wissensnetzwerke, sondern auch Machtverhältnisse und soziale Netzwerke (Hard 2003 [1990]: 376, zitiert in Aufenvenne und Steinbrink 2014).

Die Kriterien, nach denen Beiträge als Referenz für die eigene Forschung ausgewählt werden, variieren. Neben der inhaltlichen Passfähigkeit ist ein zentrales Kriterium, ob der auszuwählende Beitrag als glaubhaft bzw. wahr eingestuft wird. Ob für den Beleg einer bestimmten Aussage der erste und älteste Nachweis dieser Aussage zitiert wird, um die wissenschaftliche Priorität der Urheberin bzw. des Urhebers zu dokumentieren, oder ob eine besonders aktuelle Studie herangezogen wird, um die Gültigkeit der Aussage zu demonstrieren, bleibt den Autor*innen überlassen (Luhmann 1974: 237-238). Genauso können soziale oder strategische Kriterien eine Rolle spielen, insbesondere bei 
Zitationen einer befreundeten oder der eigenen Person. Häufig werden diejenigen Beiträge für eine Zitation ausgewählt, deren Autor*innen bereits über ein vergleichsweise hohes $\mathrm{Ma} \beta$ an Reputation verfügen (Arzheimer und Schoen 2009: 606-607). Dieses Auswahlprinzip entfaltet eine selbstverstärkende Wirkung, den sogenannten Matthäus-Effekt: Bekannte Autor*innen werden häufiger zitiert als unbekannte und werden dadurch noch bekannter (Merton 1968). Eine Ursache für diesen Effekt ist, dass angesehene Autor*innen als legitime Sprecher*innen einer bestimmten Fachgemeinschaft oder Theorie gelten können, was seitens der zitierenden Forscher*innen mitunter höher gewertet wird als die persönliche Einschätzung bezüglich der Vertrauenswürdigkeit und Originalität der Urheber*innen (Angermüller 2012: 711). Mit dem Selektionskriterium der Reputation verbunden ist außerdem ein Wahrheits- oder Gültigkeitsversprechen der zitierten Aussagen, denn sie sind stärker erprobt. Nicht zuletzt sind angesehene Wissenschaftler*innen nicht nur den Autor*innen, sondern auch der potenziellen Leserschaft eher bekannt, was der besseren Einordnung und - Passfähigkeit des Zitats vorausgesetzt - dem Verständnis der zitierten Aussage dienlich ist.

Aus der Relevanz eines wissenschaftlichen Beitrags leitet sich die Allokation von Reputation für dessen Autor*innen ab. Ein Beitrag kann folglich nur dann zu Reputation in der Fachgemeinschaft gereichen, wenn dessen Verwendung sich bei der Produktion und Veröffentlichung darauf folgender Beiträge als dienlich erweist. Umgekehrt kann - selbst neues und wahres - Wissen keine Reputation einbringen, wenn es in den anschließenden Forschungen keine Rolle spielt (Weingart 2003: 37). Die Reputation einer Akteurin bzw. eines Akteurs ist ein zentrales Kriterium für die Zuweisung von materiellen Ressourcen (bspw. Drittmittel) oder Positionen (bspw. Professuren), die wiederum für die Produktion und Publikation neuer Erkenntnisse eingesetzt werden können.

Nicht nur, weil die Rezipient*innen und Produzent*innen in Konkurrenz zueinander stehen, werden die Verwendung von Wissen und die Ermittlung von Relevanz zu einem über epistemische Kriterien hinausgehenden sozialen und politischen Prozess. Staeheli und Mitchell (2005: 357) leiten daraus einen für diese Arbeit zu berücksichtigenden Appell ab:

\footnotetext{
„[...] [R]esearch is not born relevant; it is made relevant in practice and for particular ends. Any consideration of the sociology and politics of relevance, therefore, requires consideration of why research should be relevant, how research becomes relevant, the goals of research (including political goals), and the intended audiences and beneficiaries of research. Each of these is a vexed issue; each is a locus of considerable debate as researchers with differing intellectual, social, and political agendas contend over what is right and what is best. "
} 
Der in diesem Kapitel geschilderte Mechanismus ist als idealtypisch zu verstehen und dient vor allem dazu, basale wissenschaftliche Prozesse und Kategorien für die Ableitung eines Analyserahmens (siehe Kp. 5) aufzuschlüsseln. Es ist - in umgekehrter Reihenfolge - zusammenzufassen, dass ein*e Akteur*in Reputation, materielle Ressourcen und Positionen zugeschrieben bekommt, wenn er*sie relevante wissenschaftliche Erkenntnisse auf sich verbuchen kann. Diese Relevanz manifestiert sich in der Quantität und Qualität der Bezugnahmen auf die Beiträge, die wiederum daraus resultieren, ob sich das Aufgreifen und Zitieren der Beiträge im Rahmen der jeweils eigenen Publikationen der Fachkolleg*innen als nützlich erweist. Für die Bewertung der Nützlichkeit sind nicht nur formale (Offenlegung der Quellen) und epistemische (bspw. Passfähigkeit) Kriterien ausschlaggebend, sondern auch latente bzw. soziale (Verteilung/Entzug von Anerkennung). Voraussetzung für die Rezeption eines Beitrags seitens der Fachgemeinschaft ist die Veröffentlichung von Ergebnissen, der in der Regel ein Selektions- und review-Verfahren vorausgeht. Ausgangspunkt dieses sozialen Mechanismus der Wissensproduktion ist das Formulieren und Kommunizieren einer wissenschaftlichen Erkenntnis.

\subsubsection{Epistemische Mechanismen der fachgemeinschaftlichen Erkenntnisgewinnung}

Das vorangegangene Kapitel zu den sozialen Mechanismen der fachgemeinschaftlichen Erkenntnisproduktion kam weitestgehend aus, ohne auf epistemische Mechanismen und Faktoren einzugehen. Diese heben sich im Verständnis dieser Arbeit von den sozialen Mechanismen ab, indem sie auf die Inhalte der Forschung und nicht auf deren Verwertung und Rezeption abzielen. Statt auf soziale Faktoren - Reputation, materielle Ressourcen usw. - zielen Konzeptualisierungen epistemischer Mechanismen also auf Problemwahlen, theoretische und methodische Entscheidungen, Wissensstrukturen und Forschungspfade. Wie sich im vorangegangenen Kapitel gezeigt hat und wie sich auch in diesem Kapitel zeigen wird, sind diese beiden Facetten der Wissenschaft nicht gänzlich voneinander zu trennen. So wie die Gewinnung von Erkenntnissen konditional für die Erlangung von Reputation ist, so ist die Verfügbarkeit von materiellen Ressourcen eine Voraussetzung für die weitere Forschung.

Einen überzeugenden Ansatz zur Erklärung epistemischer Praktiken und Mechanismen legen Laudel und Gläser vor (vgl. Gläser und Laudel 2015a; Gläser 2012a). Sie erforschen die Entstehung von Innovationen in der Wissenschaft sowie die Entwicklung von Forscher*innenkarrieren. Ihr Ansatz betont neben 
organisationalen Aspekten vor allem die Bedeutung der Kommunikation sowie die Wissensstruktur der Fachgemeinschaft. Sie schildern die wissenschaftliche Erkenntnisentstehung als einen dezentralen Prozess, bei dem die Einzelentscheidungen von Wissenschaftler*innen in einen Gesamtzusammenhang integriert werden. Aus den geschilderten Mechanismen der Vermittlung zwischen der kollektiven Wissensproduktion der Fachgemeinschaft und den Einzelentscheidungen ihrer Mitglieder leitet sich eine Erklärung für die Wahl von Forschungsproblemen ab.

Dabei ist zunächst konzeptionell festzuhalten, dass es in der Regel die forschende Person selbst ist, die die Entscheidungen in Bezug auf die tatsächliche Forschung und Erkenntnisgewinnung fällt. Zwar werden Probleme, Methoden und Vorgehen immer vor dem Hintergrund institutioneller Rahmenbedingungen und basierend auf dem Wissen über die Fachgemeinschaft gewählt, es sind aber dennoch die einzelnen Personen, die die Wahl treffen. Vermeintlich von Dritten (bspw. Fachkolleg*innen, Fördermittelgeber*innen) vorgegebene Forschungsentscheidungen können nicht mehr als einen Rahmen abstecken, der immer einen gewissen Gestaltungsspielraum für die Person erlaubt. Passen die strukturell vermittelten Kriterien nicht mit den eigenen Fähigkeiten und Interessen zusammen, müssen Kompromisse eingegangen oder Ausweichstrategien gefunden werden (Gläser 2012a: 12-14). Gläser (2012a: 5) veranschaulicht dies anhand der Laborstudien Knorr-Cetinas (1981), in denen sich zeigt, dass die Forschungsarbeit getragen ist von sehr vielen Einzelentscheidungen über Methoden, Geräte, Material, Kolleg*innen und andere Elemente der Wissensproduktion. Einige dieser alltäglichen Entscheidungen treffen Forschende mit expliziter Berücksichtigung möglicher Konsequenzen für den Forschungsinhalt und das Forschungsergebnis, während sie andere Entscheidungen ad hoc, ohne systematische Erwägung der Folgen für das spätere Ergebnis, treffen, ,in order to make experiments work" (Gläser 2012a: 5). Auch diese ad hoc-Entscheidungen prägen aber den Forschungsprozess und damit das produzierte Wissen.

Nichtsdestotrotz werden Forschungsentscheidungen immer vor dem Hintergrund der fachgemeinschaftlichen Wissensproduktion gefällt. Die Mitglieder einer Fachgemeinschaft identifizieren Lücken im gemeinsamen Wissen und formulieren Projekte zu deren Schließung. Alles, „was andere diesem Wissensbestand schon hinzugefügt haben oder gerade hinzufügen möchten ", ist deshalb für die individuelle Wahl des Forschungsgegenstands und die damit verbundenen Erwartungen von Bedeutung (Gläser 2012b: 151). Welche Probleme besonders dringlich und wichtig sind, wird explizit oder implizit durch die Fachgemeinschaft verhandelt und den Mitgliedern anhand von Anreizen wie bspw. der Allokation von Reputation vermittelt. Auf der anderen Seite wird die Arbeit 
zu Gegenständen abseits fachgemeinschaftlicher Prioritäten unter anderem durch Nichtbeachtung sanktioniert bzw. mit geringen Reputationschancen belegt und dementsprechend entmutigt (Luhmann 1974: 243-244).

Da die Interpretation der kollektiven Wissensproduktion von unterschiedlichen Individuen in jeweils spezifischen Kontexten parallel geschieht, ist die Passfähigkeit der einzelnen Beiträge nicht immer optimal, und verschiedene Antworten auf dasselbe Forschungsproblem sind gängig (Gläser 2012a: 6). Dennoch wird diese Form der dezentralisierten, autonomen Entscheidungsfindung bezüglich der Problemwahl für effizient befunden, da die gemeinschaftliche Wissensproduktion mit vielen Unsicherheiten behaftet ist (,welches Problem ist relevant?", ,(wie) kann es gelöst werden?', ,wer kann es am besten lösen?'). In der Regel können die Forscher*innen - zumindest nachdem sie mit Abschluss ihrer Promotion in die wissenschaftliche Unabhängigkeit entlassen wurden - selbst am besten beurteilen, welches Forschungsproblem sie in der Lage und motiviert sind zu lösen und mit welchen Mitteln sie diese Lösung anstreben. Durch die dezentralisierte Entscheidungsfindung können viele Probleme simultan formuliert und gelöst werden, sodass eine schnelle Problemlösung gewährleistet ist (Gläser 2012a: 6).

Um die Erkenntnisgewinnung einer Fachgemeinschaft, eines Individuums oder in Bezug auf einen Gegenstandsbereich zu untersuchen, modellieren Gläser und Laudel (2015a) „diachrone Wissensstrukturen“. Damit sind Netzwerke von Forschungsprojekten - hier gleichbedeutend mit Forschungsprozessen gemeint, die zwar zu unterschiedlichen Zeitpunkten ablaufen, die aber durch Input-Output-Beziehungen verbunden sind und die zentral für die Entstehung wissenschaftlicher Innovationen sind. Die Forschungsprozesse werden zum Teil durch Publikationen repräsentiert, die inhaltlich verknüpft sind und aufeinander aufbauen:

\footnotetext{
„Diachronic knowledge structures are partially represented by sets of thematically connected publications. Although researchers and thus communities hold informal knowledge as well, the published (formal) knowledge consists of those knowledge claims researchers want their community to know about and use. This publicly available knowledge can be understood as the core of a community's knowledge, and can be unobtrusively studied by bibliometrics. " (Gläser und Laudel 2015a: 303)
}

Im Zuge der kognitiven Entwicklung einzelner Forscher*innen, Arbeitsgruppen oder Fachgemeinschaften entsteht deren diachrone Wissensstruktur. Jeder Forschungsprozess speist sich aus dieser wachsenden Struktur, trägt aber gleichzeitig zu ihr bei, nährt also zukünftige Forschungsprozesse (Gläser und Laudel 2015a: 301). Für die kognitive Entwicklung von Gemeinschaften ist zu beachten, dass zwar alle Mitglieder einen Beitrag zu dieser Entwicklung leisten, 
dass die geteilte Wissensstruktur aber primär von den Langzeitmitgliedern der Gemeinschaft strukturiert wird (ebd.: 302).

Die diachronen Wissensstrukturen formen Forschungspfade (,research trails"), also Sequenzen thematisch zusammenhängender Projekte, in denen frühere Projekte als Input für spätere Projekte dienen (Chubin und Connolly 1982). Von einem Forschungspfad können sich thematisch verwandte Forschungspfade abspalten, die parallel verfolgt werden. Genauso kann ein Forschungspfad enden, wenn er nicht mehr durch weitere Projekte fortgeführt wird. Teilweise werden markante Veränderungen in der diachronen Wissensstruktur erst im Nachhinein erkennbar. Zudem geschehen Veränderungen der Forschungspfade nicht unbedingt intentional, sondern sind auch durch den Wandel äußerer Umstände bedingt (Gläser und Laudel 2015a: 320).

Das Kernargument von Gläser und Laudel ist, dass die sich verändernden Wissensstrukturen wichtige Kontextbedingungen für die Handlungen von Wissenschaftler*innen darstellen: „[...] previous research influences the choice of new research problems, as current research will - through modifying the structures - influence future choices" (Gläser und Laudel 2015a: 302). Aufgrund dieser Pfadabhängigkeit der Erkenntnisproduktion sind insbesondere die frühen Karrierephasen von Wissenschaftler*innen entscheidend für ihre spätere Selektion von Forschungsproblemen:

\begin{abstract}
„Scientific communities expect researchers to become independent during the early career phase. In this phase, many early career researchers develop long-term interests that guide their selection of projects. These interests often take the form of individual research programmes. An individual research programme can be defined as a script for future research actions that contribute to the realisation of a larger research interest, i.e. to a goal that cannot be reached by a single project. Research programmes can lead to scientific innovations if their outcomes affect the scientific community by changing the practices of many of its members. However, most of them only lead to new research trails for the researcher who designs the programme without having any farreaching community effects. " (Gläser und Laudel 2015a: 320)
\end{abstract}

Es lässt sich zusammenfassen, dass Fachgemeinschaften eine gemeinsame Wissensstruktur pflegen und erweitern, die den Kontext für die individuellen Forschungsentscheidungen von Wissenschaftler*innen darstellt. Die wissenschaftlichen Akteur*innen identifizieren Lücken in diesem Wissenskanon und formulieren Projekte zu deren Schließung. Durch das Rezipieren und Hinzufügen von Beiträgen ist die gemeinsame Wissensstruktur ständig im Wandel, allerdings unterliegt sie auch einer starken Pfadabhängigkeit, denn bestehende Forschungen bilden die Grundlage für gegenwärtige Forschungen, genauso wie gegenwärtige 
Forschungen die Wissensstruktur verändern und somit zukünftige Forschungen beeinflussen. Dies gilt es insbesondere auf der individuellen Ebene zu beachten, da Entscheidungen, die früh in der Karriere getroffen werden, den Kontext für alle weiteren Entscheidungen bilden. Hierbei ist auch auf die Betonung des Individuums hinzuweisen, welches gemäß Gläser die Wahl von Forschungsproblemen zwar vor dem Hintergrund von Wissensstrukturen und fachgemeinschaftlichen Belohnungsmechanismen, aber doch stets selbst fällt. Was die maßgeblichen Faktoren für diese Entscheidung auf individueller Ebene sind, soll im folgenden Abschnitt (Kp. 4.3) dargelegt werden.

\subsection{Der institutionelle Kontext wissenschaftlicher Akteur*innen}

Nach der Darstellung der Wissenschaft als Teilsystem der Gesellschaft, der innerwissenschaftlichen Ausdifferenzierung sowie Mechanismen der wissenschaftlichen Wissensproduktion wird nun der für wissenschaftliche Akteur*innen im deutschsprachigen Raum handlungsprägende institutionelle Kontext in den Blick genommen. Im Rahmen der hierfür gewählten institutionalistischen Forschungsperspektive wird die Wissenschaft als ein soziales System betrachtet, in dem Akteur*innen auf der Grundlage übergreifender formeller Regeln und informeller Normen individuell handeln. Der institutionelle Kontext prägt Akteur*innen, indem er Handlungsressourcen und Handlungsorientierungen sowie die innere Organisation komplexer Akteur*innen strukturiert. Er konstituiert Entscheidungssituationen, indem er die Entscheidungsregeln bestimmt, die beteiligten Akteur*innen benennt, ihnen Handlungsoptionen vorgibt und diese mit Anreizen versieht (Treib 2015: 280). Es werden im Folgenden insbesondere drei Einflussgrößen des institutionellen Rahmens - formale Koordination, Allokation von Reputation, Allokation von materiellen Ressourcen - erläutert.

Indem auf die Handlungsbedingungen fokussiert wird, lässt sich die im Folgenden dargelegte institutionalistische Perspektive am ehesten der Mesoebene zuordnen, denn es werden organisationale Mechanismen, Interdependenzen zwischen Akteur*innen sowie die Bewältigung dieser Interdependenzen betont, um die abstrahierten Entscheidungsbedingungen auf Akteur*innenebene zu erklären. Im Hinblick auf die zu Anfang des Kapitels aufgeworfenen Fragestellungen ,Wie wählen wissenschaftliche Akteur*innen ein Thema?' bzw. ,Wie lassen sich Veränderungen der aggregierten Entscheidungen wissenschaftlicher Akteur*innen hinsichtlich ihrer Themenwahl erklären?' - stellt dieser Ansatz die finale Konkretisierung dar, sodass dieses Teilkapitel eine zentrale Funktion für die Ableitung 
des Analyserahmens im darauffolgenden Abschnitt leistet. Aufgrund der daraus resultierenden hohen Passfähigkeit dieses Teilkapitels mit der anschließend zu entwickelnden Heuristik ist klarzustellen, dass die hier (Kp. 4.3) erfolgenden Schilderungen, so wie alle bisherigen Theoriekapitel, eine fokussierte Aufarbeitung und Systematisierung des Forschungsstandes darstellen. Hiervon abzugrenzen ist die Anpassung und Aneignung ausgewählter Aspekte im Hinblick auf die Bearbeitung der vorliegenden Fragestellung, die in Kapitel 5 erfolgt.

Seit der Kuhn'schen Wende in der Wissenschaftssoziologie rücken individuelle Handlungsziele, wie bspw. das Akkumulieren von Reputation oder materiellen Ressourcen, in den Vordergrund institutionalistischer Untersuchungen, während ethische Normen und Ideale, wie bspw. das Streben nach Wahrheit, (wenn überhaupt) als Mittel zum Erreichen der Handlungsziele eingestuft werden (Schimank 2010). Im Rahmen dieser Tradition identifizieren Langer (2006), Braun (2001) und Hüther et al. (2010) jeweils vier oder fünf Kapitalarten, nach denen Wissenschaftler*innen streben (Braun 2001: 245). In der Synopse lassen sie sich zu drei Kapitalarten zusammenfassen: reputatives, ökonomisches und formales bzw. politisches Kapital. Braun nennt auch das erzieherische Kapital, das aber erstens nur eine geringe Rolle im deutschen Hochschulsystem spielt und zweitens keinen Erklärungsgehalt für die Wahl von Forschungsthemen bietet (ebd.). Schimank (2014: 34-35) erkennt ähnliche Handlungslogiken, kommt aber zu einer Einteilung in fünf „Stellschrauben“, mittels derer Einfluss auf das Forschungshandeln einer Person ausgeübt wird: Formale Weisungen, materielle Ressourcen, Zeit, Karriere und Reputation. Basierend auf Schimanks Arbeiten nehmen wiederum Aljets und Lettkemann (2012) eine eigene Unterteilung in drei „Einflusskanäle“ vor: „Allokation von Ressourcen“2, „Allokation von Reputation“ und „Formale Koordination". Sie fassen dabei die Faktoren Zeit, die Schimank letztlich als „manpower" verzeichnet und die sich bspw. durch Veränderung des Lehrdeputats oder des Personalstocks ergibt, und Karriere, unter die Schimank insbesondere Rekrutierungsentscheidungen subsummiert, unter dem Faktor materielle Ressourcen zusammen (Schimank 2014: 34 f.; Aljets und Lettkemann 2012: 132 ff.). Resümierend kann gesagt werden, dass die verschiedenen Schilderungen des Handlungskontextes der Wissenschaft nahezu kongruent sind und Unterschiede primär in der Schwerpunktsetzung, Systematisierung und Nomenklatur auftreten.

\footnotetext{
${ }^{2}$ Zur Vermeidung von Missverständnissen sei angemerkt, dass Schimank, Aljets und Lettkemann den Begriff ,Ressourcen ' als synonym mit ,materiellen Ressourcen 'oder ,ökonomischem Kapital ' verwenden. Der in dieser Arbeit verwendete Ressourcenbegriff ist, mit Ausnahme der Verwendung in diesem Zitat, jedoch breiter gefasst und bezieht bspw. auch Sozialkapital mit ein. Er basiert auf dem Ansatz des Akteurzentrierten Institutionalismus (siehe Kp. 5.2; vgl. Mayntz und Scharpf 1995: 54; Scharpf 2006: 86-87).
} 
Die folgende Systematisierung in drei grundsätzliche Einflussgrößen des institutionellen Kontextes folgt im Wesentlichen der von Aljets und Lettkemann, die insbesondere von Aljets (2015: 82-106) vertieft werden. Demnach bilden die formale Koordination (Kp. 4.3.1), die Allokation von Reputation (Kp. 4.3.2) und die Allokation von materiellen Ressourcen (4.3.3) die institutionellen Rahmenbedingungen, die für das Handeln von Wissenschaftler*innen maßgeblich sind (Aljets und Lettkemann 2012). Es handelt sich bei den einzelnen Einflussgrößen um analytische Konstruktionen, die nicht immer trennscharf verlaufen und einander bedingen. Letzteres wird insbesondere in dem abschließenden Teilkapitel (4.3.4) verdeutlicht, das die drei Einflussgrößen miteinander in Beziehung setzt.

\subsubsection{Formale Koordination}

Der Begriff der formalen Koordination umfasst gemäß Aljets (2015: 101) die auf Hierarchie basierenden Koordinationsmechanismen, die formalen Entscheidungswege sowie die rechtlichen Rahmenbedingungen, die das Forschungshandeln wissenschaftlicher Akteur*innen strukturieren. Durch formale Koordination werden nicht nur Verfahrenswege vorgegeben, sondern auch die zu beteiligenden Akteur*innen benannt sowie deren Rechte und Kompetenzen definiert. Auf der anderen Seite werden Akteur*innen, die bestimmte formelle Kriterien nicht erfüllen, von Organisationen oder Plattformen ausgeschlossen. Allerdings geht es in den allermeisten formal strukturierten Handlungssituationen, so Aljets mit Verweis auf Luhmanns (1964) Erwartungsstruktur, weniger um die tatsächliche formale Anweisung konkreter Handlungen: ,Vielmehr strukturiert bereits die Möglichkeit, dass ein Akteur eine Handlung formal anweisen könnte, die Handlungskoordination zwischen den Akteuren maßgeblich" (Aljets 2015: 101; siehe auch Hüther et al. 2010: 172-176).

Die formale Koordination der Wissenschaft ist durch verschiedene Governance-Mechanismen konstituiert, die einen Teil der Handlungsbedingungen wissenschaftlicher Akteur*innen determinieren. Gemäß einer weit verbreiteten Typologie, die auf Schimank und Meier (2002) sowie Braun und Merrien (1999) zurückgeht, lassen sich fünf formale Governance-Mechanismen in der Wissenschaft unterscheiden: Staatliche Regulierung, Außensteuerung, hierarchische Selbststeuerung, akademische Selbstorganisation und Konkurrenz (siehe insb. Hüther und Krücken 2016: 136-144). Dabei bezeichnet ,Staatliche Regulierung ' die hierarchische Steuerung durch bürokratische Vorgaben, etwa im Bereich des Personalrechts oder der Mittelzuwendung. Der Begriff, Außensteuerung ' verweist 
ebenfalls auf die Einflussnahme durch den Staat, allerdings nicht in Form detaillierter Anweisungen, sondern in Form von Zielvorgaben, sodass die Wege der Zielerreichung in der Hand der Wissenschaft liegen. Der Außensteuerungsmechanismus beinhaltet die Einsetzung und Beteiligung intermediärer Organisationen und Governance-Institutionen, wie bspw. Akkreditierungsagenturen oder Hochschulräte. Bei dieser Form der Koordination können wiederum hierarchische Elemente zum Tragen kommen, häufiger findet allerdings eine Ersetzung der Hierarchie durch eine Koordination über Verhandlungen statt. Zur Außensteuerung bemerken Hüther und Krücken (2016: 138), dass durch die Übertragung hierarchischer Kompetenzen an die intermediären Organisationen das Kontrollund das Informationsproblem hierarchischer Koordination minimiert werden:

\begin{abstract}
„Dies geschieht erstens dadurch, dass intermediäre Einheiten als Teil der Hochschulen konzipiert sind (zum Beispiel einige Hochschulräte) und dabei angenommen wird, dass hierdurch eine höhere Kontroll- und Informationsdichte möglich ist. Zweitens finden wir bei anderen intermediären Einheiten eine hohe Spezialisierung (zum Beispiel Akkreditierungsagenturen), die zumindest das Informationsproblem dadurch minimiert, dass durch die Spezialisierung die Akkumulation von Wissen und Informationen zu einem bestimmten Gegenstandsbereich erleichtert wird. “
\end{abstract}

Unter ,hierarchischer Selbststeuerung ' werden die hochschul- und institutsinternen Steuerungsmechanismen gefasst, bei denen die Leitungsebene Entscheidungen vornimmt und diese durchsetzt. Dagegen bezieht sich die ,akademische Selbstorganisation' auf die formale Koordinierung seitens der Entscheidungsbetroffenen (Wissenschaftler*innen) selbst im Rahmen der von ihnen dominierten Gremienstrukturen. Die Entscheidungen der Gremien beruhen dabei im Normalfall nicht auf Hierarchie, sondern auf Verhandlung und anschließendem Mehrheitsentscheid (Hüther et al. 2010: 119-120; Schimank und Meier 2002: 5). Der Mechanismus der ,Konkurrenz' bezieht sich auf die im Zuge der Etablierung von New Public Management-Ansätzen verstärkte Abhängigkeit der Ressourcenversorgung wissenschaftlicher Akteur*innen von der relativen Leistungsfähigkeit der Organisationseinheiten bzw. auf die diesen Mechanismus vermittelnden Regeln, die sich in Form zunehmender Einflussnahmen durch Evaluationen und der Einschränkung dauerhafter Berufungs- und Bleibezusagen manifestieren (Schimank und Meier 2002: 5). Zusätzlich zu den fünf geschilderten Mechanismen sind auch nicht-staatliche, wissenschaftsexterne Akteur*innen zu berücksichtigen, die teilweise ebenfalls die formale Koordination der Wissenschaft konstituieren, bspw. in Form von Stiftungsprofessuren.

Im Zuge der sogenannten New Public Management Reformen haben sich seit den späten 1990er Jahren einige institutionelle Regelungsstrukturen, insbesondere 
an den Hochschulen, gewandelt. Dabei wurde einerseits die universitäre Leitungsebene gestärkt und andererseits die direkte staatliche Regulierung zugunsten einer gesteigerten Output-Orientierung reduziert. Insbesondere Mechanismen der leistungsorientierten Mittelvergabe und der Einsatz von Zielvereinbarungen wurden mehr und mehr eingesetzt. Im Zuge dessen haben intermediäre Organisationsformen (bspw. Hochschulräte) sowie die (gegenseitige) Koordinierungsfunktion wissenschaftlicher Eliten (bspw. im Rahmen von Evaluierungskommissionen) an Bedeutung gewonnen. In der Folge wurden hierarchische Strukturen zunehmend durch netzwerkartige und marktförmige Koordinationsmechanismen (bzw. bei Schimank und Meier Mechanismen der, Außensteuerung ' und ,Konkurrenz') ersetzt, sodass die Interdependenzen zwischen wissenschaftlichen Akteur*innen, und dabei insbesondere jene zwischen den wissenschaftlichen Eliten, zugenommen haben (Aljets 2015: 101-105; Hüther und Krücken 2016: 50-61).

Durch formale Koordination wird von verschiedenen Akteur*innen auf verschiedenen Ebenen Einfluss auf die Wissensproduktion genommen. Im Rahmen hierarchischer Beziehungen (bspw. Ministerium gegenüber Fakultät, Fakultät gegenüber Lehrstuhlinhaber*in, Lehrstuhlinhaber*in gegenüber Mitarbeiter*in) werden direkte Anweisungen sowie Handlungsziele definiert und durchgesetzt. Auf die Organisationsebene (bspw. Fakultäten und Forschungsinstitute) wird Einfluss bspw. durch Mechanismen der leistungsorientierten Mittelvergabe sowie durch Evaluationsverfahren genommen. Diese von wissenschaftsexternen Akteur*innen ausgehenden und teilweise durch intermediäre Instanzen vermittelten Beeinflussungsversuche werden wiederum auf die Ebene untergeordneter Organisationen (bspw. Lehrstühle) sowie letztlich der individuellen Forscher*innen in der Form formaler Weisungen und Zielvorgaben weitergegeben. Ein Beispiel hierfür sind Berufungsverfahren, bei denen zunächst in der Ausschreibung Auswahlkriterien formuliert und in den Verhandlungen Zielvorgaben vereinbart werden, deren Nichterreichung sanktioniert wird (Gläser 2012a: 9). Bereits die Erlangung der sogenannten ,Berufungsfähigkeit‘ ist mit impliziten und expliziten Vorgaben verbunden, wie bspw. einer abgeschlossenen Promotion oder dem Nachweis des wissenschaftlichen Impacts anhand von Zitationsindizes. Die Inhalte der Forschung lassen sich insofern durch formale Koordination beeinflussen, als dass im Rahmen hierarchischer Beziehungen die Bearbeitung bestimmter Inhalte angeordnet werden kann, bspw. im Rahmen von Angestelltenverhältnissen oder durch die Denomination von Stellen. Auch durch quantitative Zielvorgaben kann der Inhalt beeinflusst werden, indem deren Erreichung bspw. mit der Wahl möglichst ertragreicher Forschungsmethoden, -kooperationen oder -themen zusammenhängt. 
Aus der formalen Koordination - bzw. aus den formalisierten Positionen, Funktionen, akademischen Graden, Mitgliedschaften u.ä. - leiten sich individuelle Handlungsressourcen für die wissenschaftlichen Akteur*innen ab. Akteur*innen werden mit Rechten, Kompetenzen und einem Einflussbereich ausgestattet, sie werden aber gleichzeitig mit Verpflichtungen belegt, und ihre Handlungsspielräume werden eingegrenzt. Diese auf formaler Koordination basierenden Ressourcen werden auch als formales und politisches Kapital, institutionelle Macht oder Einfluss gefasst (Langer 2006: 46-62; Hüther et al. 2010: 179-182). Sie lassen sich auf verschiedenen Wegen in andere Handlungsressourcen, eintauschen'. So erleichtert bzw. ermöglicht eine hochrangige Position den Zugang zu materiellen Ressourcen und die Aufnahme persönlicher Bekanntschaften zu wiederum einflussreichen Akteur*innen. Zudem ist zur Erlangung hochrangiger Positionen, neben der Erfüllung gewisser formaler Mindestanforderungen, vor allem das Erlangen einer gewissen Reputation Voraussetzung. Im Umkehrschluss indiziert eine hochrangige Position, dass der*die Innehaber*in in der Vergangenheit Reputation angehäuft hat, sodass die Bekleidung der Position für sich schon Ansehen verleiht. Diese in Positionen geronnene Reputation stellt nicht nur für die Fachgemeinschaft eine Strukturierungshilfe dar, sondern auch die gesellschaftliche Umwelt kann anhand der Reputation besondere Leistungen und herausgehobene Akteur*innen erkennen und auf dieser Grundlage Anknüpfungspunkte erschließen (Luhmann 1974: 237-238). Allerdings steht das Bekleiden von Positionen bzw. der Aufbau politischen Kapitals gleichzeitig in einem Spannungsverhältnis zur Erlangung von Reputation, da beide Kapitalarten nur mit hohem zeitlichen Aufwand und unter großen Anstrengungen zu erreichen sind (Hüther et al. 2010: 168-169).

Langer (2006: 47-48) sowie Hüther et al. (2010: 179-182) betonen die Bedeutung des „mikropolitischen Kapitals“ wissenschaftlicher Akteur*innen, also des Wissens und der Fähigkeiten, die benötigt werden, um die formellen Regeln und Mechanismen überhaupt aktivieren und zum eigenen Vorteil einsetzen zu können. Es besteht aus zwei Komponenten:

„(a) Wissen über politisch-historische Hintergründe aktueller Entscheidungsprobleme, etwa Berufungszusagen, Themenkarrieren, die Entstehungsgeschichte relevanter Beschlusslagen, Präzedenzfälle etc., damit auch über aktuelle ,informelle Strukturen', Rechtslagen, Verfahrensfragen, Finanzierungsmöglichkeiten, mögliche Handlungsalternativen etc.

(b) Akkumulierte Kompetenzen und Ressourcen zur politischen Durchsetzung und Gestaltung universitärer Organisationsstrukturen und die Bereitschaft und Fähigkeit, sie für den Einsatz in den Auseinandersetzungen und Entscheidungsprozessen der Selbstverwaltungsorgane symbolisch zu bündeln. " (Langer 2006: 47) 
Zu den akkumulierten Kompetenzen und Ressourcen zählen wiederum Verhandlungsgeschick, strategisch-taktisches Können, sowie das Aufbauen, Pflegen und Einsetzen-Können nützlicher Beziehungen (Langer 2006: 47-48). Hüther et al. (2010: 181) heben die Bedeutung von Koalitionen und Netzwerken hervor, in denen informell und häufig nicht sichtbar Einfluss auf die formellen Entscheidungsprozesse geübt werden kann. Um derartige Bündnisse eingehen zu können, muss man „etwas anzubieten haben“:

„Um mikropolitisches Kapital zu generieren, ist eine gegenwärtige oder vergangene Besetzung von Positionen mit formale [m] Kapital besonders vorteilhaft. Zunächst können Positionsinhaber Einfluss innerhalb des Netzwerkes anbieten und koordinieren. Zudem verfügen diese über Informationen und Wissen, das gleichfalls innerhalb des Netzwerkes genutzt werden kann. Neben Positionen stellt aber auch reputatives Kapital von Professoren eine Möglichkeit dar, in Netzwerke integriert zu werden. Professoren mit hoher Reputation können so insbesondere die Legitimität der informell getroffenen Entscheidungen gegenüber Netzwerkexternen befördern. "(Hüther et al. 2010: 181)

Die Beteiligung an mikropolitischen Bündnissen ist nicht „kostenfrei“, sondern es kommt im Zuge wiederholter gegenseitiger Unterstützungen und Absprachen zu einer ,Verkettung von Verpflichtungen“, die wiederum Machtpotentiale schafft (Hüther et al. 2010: 181-182).

Als weitere Prozesse der informellen Anwendung und Ausgestaltung formaler Regeln sind die vorherrschende Institutskultur sowie persönliche Interaktionsverhältnisse zu beachten. Am Beispiel des Machtverhältnisses zwischen Professor*in und Mitarbeiter*in legt Langer (2006: 61-63) dar, dass häufig auf der Basis gegenseitigen Vertrauens agiert wird, sodass das formal festgeschriebene Abhängigkeitsverhältnis nicht oder kaum als solches in Erscheinung tritt. Erkennbar werden formale Abhängigkeiten insbesondere erst bei Meinungsverschiedenheiten oder unterschiedlichen Interessenlagen. Gegebenenfalls können Weisungsbefugte nicht nur mit informellen Sanktionen, wie bspw. geringschätziger Behandlung, reagieren, sondern auch auf ihre formale Macht zurückgreifen, bspw. indem sie unerwünschte Aufgaben erteilen oder im Extremfall die Promotion oder Habilitation eines Mitarbeiters bzw. einer Mitarbeiterin hintertreiben (ebd.). 


\subsubsection{Allokation von Reputation}

Reputation gilt gemeinhin als grundlegendes Strukturierungselement der Wissenschaft und Reputationsstreben als die zentrale Handlungsorientierung von Wissenschaftler*innen (Luhmann 1974; Whitley 1984; Schimank 2010). Sie lässt sich als Bekanntheit und fachliches Ansehen einzelner Wissenschaftler*innen oder Forschungsgruppen innerhalb der wissenschaftlichen Gemeinschaft beschreiben, umfasst aber auch das Ansehen von Medien, Organisationen und Institutionen, wie bspw. Zeitschriften, Verlagen, Instituten, Universitäten oder Tagungen (Luhmann 1974: 237; Langer 2006: 42). Gemäß Weingart (2003: 22-23) signalisiert die Reputation eines*einer Wissenschaftler*in ,sowohl innerhalb des Systems als auch nach außen, dass es sich um jemanden handelt, der einen wichtigen und von seinen Kollegen als solchen anerkannten Beitrag geleistet hat".

Luhmann (1974: 237) beschreibt Reputation als eine Art unbefristeten Kredit, der sich auf Grundlage der gezeigten Einzelleistungen bildet. Wer darüber verfügt, kann einem Beitrag allein durch seine Autorenschaft Aufmerksamkeit verleihen, weil bestimmte Wissenschaftler*innen sich einen (unterschiedlich gearteten) Nutzen von dessen Rezeption versprechen. Dieses Versprechen muss dann allerdings auch konstant eingelöst werden, das Anspruchsniveau muss also gehalten werden, denn die Beiträge stehen unter besonderer Beobachtung (Luhmann 1992: 245-248). An der Wichtigkeit von Namen in der Wissenschaft lässt sich diese zentrale Funktion der Reputation ablesen. So enthalten bspw. Bücher oft Namensverzeichnisse, damit Aussagen zu bzw. von bestimmten Akteur*innen ohne Lesen des Buches rasch gefunden werden können. Für Luhmann gibt diese Fokussierung auf Namen Anlass dazu, das Bild der Wissenschaft als primär erkenntnismotivierte Gemeinschaft generell in Frage zu stellen:

„Aber wozu muss man wissen, wer die Wahrheit ans Licht gebracht hat, wenn man sich doch direkt an die Wahrheit halten und ihren Urheber ohne Schaden vergessen kann?" (Luhmann 1992: 245-248).

Die Zuschreibung von Reputation erfolgt überwiegend dezentral und in einem „eher diffusen Prozess“ (Gläser 2012b: 157). Sie resultiert primär aus der Rezeption der wissenschaftlichen Beiträge von Akteur*innen durch die Fachgemeinschaft bzw. durch die Kommunikation darüber. Sie ist folglich das Ergebnis alltäglicher Entscheidungen wissenschaftlicher Akteur*innen darüber, welche bzw. wessen Beiträge besprochen, weiterverwendet und zitiert werden und woran sich die jeweilige Forschung orientiert. Dabei ist nicht nur entscheidend, wie viele Mitglieder der Fachgemeinschaft über den Beitrag kommunizieren, sondern auch 
mit welcher Qualifizierung (befürwortend oder ablehnend), mit welchem Stellenwert (zentral oder als Randnotiz) und in welchem Medium (in einem angesehenen Fachjournal oder im Gespräch) die Kommunikation erfolgt. Auch durch welche Akteur*innen (mehr oder weniger angesehen in der Fachgemeinschaft) die Kommunikation erfolgt, ist maßgeblich, denn es sind hauptsächlich die Eliten, die für die Reputationszuschreibung verantwortlich sind (Aljets 2015: 93-96).

Die Dezentralität der Reputationsallokation bedingt, dass die Zuschreibung der Reputation erst nach der Veröffentlichung erfolgen kann und nicht planbar ist, auch wenn Faktoren identifiziert werden können, die die Aussicht auf Reputation erhöhen, wie bspw. die Reputation der Autor*innen oder die Größe der angesprochenen Zielgruppe. Es besteht also eine Unsicherheit bei der Produktion und Veröffentlichung von Forschungsergebnissen in Bezug auf den daraus folgenden Reputationserwerb. Aus den allermeisten wissenschaftlichen Publikationen geht keine durch Zitierung dokumentierte Reputationszuschreibung hervor (Aljets 2015: 93-96).

Neben der Veröffentlichung von Beiträgen in Publikationen oder Vorträgen gibt es weitere Quellen für den Erwerb von Reputation. So steigert das Einwerben von Drittmitteln das Ansehen, wobei auch hier nicht nur die Quantität, sondern bspw. auch das Format der Forschung (Grundlagenforschung oder angewandte Forschung) oder die Fördermittelgeber*innen (bspw. DFG oder Wirtschaftsunternehmen) maßgeblich sein können (Hüther et al. 2010: 177-178). Ist mit einem Drittmittelprojekt eine Begutachtung auf Grundlage wissenschaftlicher Kriterien verbunden, kann anhand der Einwerbung abgelesen werden, dass ein*e Akteur*in (weiterhin) innovative und relevante Forschung betreibt. Indem Drittmitteleinwerbung ein wesentliches Ziel von Forschungseinrichtungen ist und wissenschaftliche Akteur*innen in einen Wettbewerb um diese (knappen) Mittel treten, signalisiert deren Einwerbung Erfolg in der Wissenschaft. Umgekehrt ist für die Bewilligung eines Fördermittelantrags in der Regel auch die Reputation oder zumindest die durch einschlägige Vorarbeiten erlangte persönliche Eignung der Antragsteller*innen förderlich (ebd.: 178).

Neben dem Einwerben von Drittmitteln, verspricht auch die Begutachtung der Drittmittelanträge und -projekte Reputation, wie Aljets (2015: 99) schließt:

„Die gestiegene Bedeutung von Gutachtertätigkeiten in der wissenschaftlichen Wahrnehmung lässt sich beispielsweise an den Auflistungen dieser Tätigkeiten in Wissenschaftlerlebensläufen beobachten. Dabei wird davon ausgegangen, dass allein durch die Tätigkeit als Gutachter bereits Reputation zugeschrieben wird. Diesem Reputationsmechanismus liegt die Annahme zu Grunde, dass nur diejenigen für Gutachtertätigkeiten in Frage kommen, die in ihrem Wissenschaftsgebiet besondere 
Leistungen oder Kenntnisse vorweisen können. Die Gutachtertätigkeit bestätigt damit den fachlichen Status des Gutachters. “

Auch das Bekleiden von leitenden Funktionen, bspw. in Fachgesellschaften oder Forschungsinstituten, kann zu einer Reputationsallokation in beide Richtungen führen, denn nicht nur Ämter können Personen Ansehen verleihen, sondern auch umgekehrt können Personen Ämtern Reputation verschaffen (Hüther et al. 2010: 170). Luhmann (1974: 237) spricht in diesem Kontext von „Ansteckung“, denn Reputation lasse sich übertragen, ,indem renommierte Autoren den Ruf einer Zeitschrift aufbessern, renommierte Professoren den Ruf einer Fakultät oder umgekehrt". Auch Beziehungen zu angesehenen Wissenschaftler*innen, dokumentiert bspw. durch Ko-Autorenschaft oder gemeinsame Forschungsprojekte, können zur Übertragung von Reputation führen. Die Einbettung einer Person in das Netzwerk der wissenschaftlichen Community ist folglich nicht nur Ausdruck und Folge ihrer Reputation, sondern umgekehrt konstituiert sich die Reputation einer Person auch durch ihre Netzwerkposition. Auch weiteres Hintergrundwissen über die Karriere einer Person, etwa zu ihren Ausbildungs- und Karrierestationen, kann eine Rolle für die Zuschreibung von Reputation spielen (Angermüller 2012: 711). Zudem kann die Beteiligung an öffentlichen Diskussionen, bspw. durch Beiträge in Tageszeitungen oder Interviews für Fernsehen, Radio oder Internet, die Reputation fördern, teilweise aber auch beeinträchtigen (Hüther et al. 2010: 170).

Die Reputationszuschreibung erfolgt größtenteils kumulativ, was sich darin zeigt, dass das Ausbleiben von Beiträgen nicht zum sofortigen Verlust der bis dahin gewonnenen Reputation führt. Auch können Wissenschaftler*innen Risiken eingehen, ,ohne im Falle eines Misserfolges die umfassende Diskreditierung fürchten zu müssen " (Gläser 2012b: 157). In diesem Kontext hebt Luhmann (1974: 238) die besondere Elastizität der Reputation hervor:

„Sie erlaubt es, Namen und Ansehen einzelner Personen oder Einrichtungen mit spezifischen Leistungen fest zu verbinden, ohne dass dies der Austeilung neuer Reputationen für andere, vielleicht sogar entgegengesetzte Leistungen entgegenstünde. Reputation ist zwar knapp, weil Aufmerksamkeit knapp ist, wird aber nicht wie im Falle von Geld, formalisierter Macht oder Energie durch ein Summenkonstanzprinzip reguliert. Alte Reputation braucht deshalb bei Neuausteilungen nicht schmerzhaft entzogen zu werden, sie verblasst nur im Vergleich mit der neuen Reputation und wird auf unmerkliche Weise geschichtlich. Aus diesen Gründen ist Reputation ein innovationsgünstiges Medium, und dies um so mehr, je prompter sie der Leistung folgt, je rascher sie im System fließt. ,Revolutionen' in der Wissenschaft sind, obwohl es in ihnen doch um Wahrheit geht, mit erstaunlich geringen sozialen Konflikten belastet. “ 
Dennoch kann die Reputation von Wissenschaftler*innen auch sinken. Besonders unmittelbar und empfindlich reagiert Reputation auf diskreditierende Information (Luhmann 1974: 239). Mittel- bis langfristig ist die Reputation einer Person dadurch gefährdet, dass die Bedeutung ihrer Beiträge sinkt bzw. die Kommunikation über die Beiträge nach und nach entfällt. So kann die Berufung seitens der Fachgemeinschaft auf anfänglich relevante Erkenntnisse obsolet werden, sobald sich andere Ideen durchsetzen, ob in Form einer grundsätzlichen Neuorientierung (Paradigmenwechsel) oder durch eine marginale Modifikation, in der die Fachgemeinschaft eine Verbesserung erkennt, etwa bezüglich der Verständlichkeit oder der Präzision des Beitrags. Zudem kann ein Wandel der Agenda die Relevanz einer Person unterminieren, wenn diese ihre Reputation primär aus Beiträgen zu einem Thema zieht, von dem sich aber ein großer Teil der Fachgemeinschaft zugunsten anderer Themen abwendet.

Für die Wissenschaftler*innen bietet die Reputation zunächst eine Strukturierungshilfe, um angesichts der Komplexität heutiger Wissensproduktion den Überblick zu behalten. Da es selbst in stark spezialisierten Kommunikationsgemeinschaften nicht möglich ist alle Fachbeiträge zu lesen, wählen wissenschaftliche Akteur*innen die Reputation von Autor*innen und Beiträgen als zentrales Orientierungskriterium, um aus der Masse an Veröffentlichungen eine Auswahl für die Kenntnisnahme und Verwendung von Beiträgen treffen zu können (Aljets 2015: 93-96; Weingart 2003: 25-26). Das Grundprinzip hierbei ist, dass vergangene Leistungen im Rahmen der wissenschaftlichen Erkenntnisproduktion sowie eine hervorgehobene Stellung in der fachgemeinschaftlichen Kommunikation sich in der Reputation einer Person manifestieren. Damit einhergehend wird die Reputation einer Person für die Fachkolleg*innen zum Indiz für zukünftig zu erwartende Leistungen sowie zum Indikator dafür, ob die Kenntnisnahme und Zitierung eines Beitrags dem eigenen Erkenntnisprozess sowie dem eigenen Ansehen zugutekommen oder nicht (Weingart 2003: 25-26).

Für das System Wissenschaft nimmt die Reputation darüber hinaus die Funktion eines Vermittlungsmediums zwischen der akademischen Wissensproduktion und dem System für Verteilungsentscheidungen ein (Luhmann 1974: 237-238). Indem über die Allokation von Posten und materiellen Ressourcen unter anderem auf Grundlage der Reputation entschieden wird und indem der Erwerb dieser Reputation primär an die Produktion neuen Wissens geknüpft ist, wird Wissensproduktion zu einer - über Reputation vermittelten - Bedingung für das Erlangen von Posten und Ressourcen (ebd.). Der Reputationsmechanismus gilt daher als Motor für den wissenschaftlichen Erkenntnisfortschritt, der auch mit der inhaltlichen Ausrichtung der Wissenschaft verknüpft ist, so Gläser (2012b: 157): 
„Da man mit der Lösung von für den weiteren Erkenntnisfortschritt besonders wichtigen Problemen besonders viel Reputation erwerben kann, werden mehr Wissenschaftler sich hier versuchen, was wiederum die Wahrscheinlichkeit des Gelingens erhöht. "

Im Gegensatz zur Qualität ihrer Beiträge ist die Reputation von Wissenschaftler*innen auch für Akteur*innen außerhalb der Fachgemeinschaft (wenn auch mit Einschränkungen) lesbar. Dabei nehmen insbesondere quantitative Indikatoren der Leistungskontrolle eine zunehmende Bedeutung ein, da sie es auch Akteur*innen ohne einschlägige wissenschaftliche Expertise ermöglichen, die Leistungen von Wissenschaftler*innen zu vergleichen. Quantitative Reputations- und Leistungsvergleiche sind daher besonders attraktiv für diejenigen Akteur*innen, die die Forschungsleistung einer bestimmten Person inhaltlich nicht nachvollziehen können, weil sie nicht der gleichen Fachgemeinschaft bzw. nicht der Wissenschaft angehören (Aljets 2015: 93-96).

Die reine Quantität von Drittmitteln, Publikationen und Zitationen, die Wissenschaftler*innen, Organisationen oder Medien auf sich verbuchen können, gewinnt auch innerhalb der Wissenschaft in den vergangenen Jahren an Bedeutung. Bei Karriereentscheidungen wie Berufung oder Entfristung, bei Förderentscheidungen oder bei Evaluationen ist die quantifizierende Leistungserfassung, etwa durch Impact-Faktoren, H-Indizes oder Drittmittelquoten, zu einem zentralen Argument geworden (Schimank 2010: 234-240; Aljets 2015: 93-96). Die quantitativen Daten lassen sich mit relativ geringem Aufwand ermitteln, und sie haben gegenüber subjektiven Einzelbewertungen den Vorteil der Objektivität, auch wenn die Auswahl und Bedeutung der Indikatoren, die Datengrundlage und die Berechnung weiterhin streitbar sind.

Zwar sind die quantitativen Kennziffern primär ein innerwissenschaftliches Produkt, indem bspw. Zitationskennziffern sich nur aus Bezugnahmen der wissenschaftlichen Gemeinschaft speisen (sollten) und indem über Veröffentlichungen in Fachzeitschriften oder die Allokation bestimmter Drittmittel Fachkolleg*innen (mit-)entscheiden (peer review). Die Berechnung, weitere Verwendung und Interpretation dieser Daten entzieht sich jedoch zunehmend der Kontrolle der Fachgemeinschaft. In dieser mehr und mehr systemextern gesteuerten Beimessung wissenschaftlicher Reputation erkennt Schimank (2010: 239) einen Verlust an Autonomie oder ,occupational control“, zumal wissenschaftliche Reputation traditionell als Ausdruck der Autonomie der Wissenschaft galt. Gemäß Aljets (2015: 94-95) lassen sich einige „Dysfunktionalitäten“ in der wissenschaftlichen Erkenntnisproduktion diesen Entwicklungen zuschreiben, darunter die steigenden Betrugsfälle, die Reduktion der vielseitigen wissenschaftlichen 
Tätigkeit auf das Publizieren und die Drittmitteleinwerbung, die Verengung der individuellen Forschungs-Portfolios sowie die Förderung eines wissenschaftlichen „Mainstreams“.

\subsubsection{Allokation von materiellen Ressourcen}

Die materiellen Ressourcen wissenschaftlicher Akteur*innen oder auch ihr ökonomisches Kapital umfassen Personalmittel und Sachmittel. Zu den Sachmitteln sind unter anderem ein Arbeitsplatz, technische Ausstattung, Zugang zu einer Bibliothek oder Reisemittel zu zählen. Aljets (2015: 83) zählt mit Verweis auf Schimank (2014: 34) weiterhin den Faktor Zeit zu den materiellen Ressourcen, wobei darunter zur Forschung verfügbare Arbeitszeit bzw. „manpower “ gefasst wird, die durch das zugeordnete Forschungspersonal, aber bspw. auch durch die Höhe des Lehrdeputats bestimmt wird.

Das ökonomische Kapital wird hauptsächlich von staatlichen Ministerien, Forschungs- und Förderorganisationen sowie von Wirtschaftsunternehmen in Form von Grundmitteln und Drittmitteln bereitgestellt. Mit Grundmitteln werden die durch die Unterhaltsträger*innen zur Verfügung gestellten laufenden Haushaltsmittel und Investitionen von Wissenschaftler*innen, Lehrstühlen, Instituten oder Hochschulen verstanden, wobei in dieser Arbeit der auf die Forschung verwendete Teil der Sach- und Personalmittel interessiert. Der Begriff Drittmittel umfasst Gelder, die zusätzlich zu diesen Grundmitteln von öffentlichen oder privaten Stellen bereitgestellt werden. Sie sind in der Regel an ein konkretes Projekt bzw. einen konkreten Zweck gebunden, meist zeitlich begrenzt und werden häufig in einem kompetitiven Verfahren von einzelnen Forscher*innen, Fachbereichen, Instituten oder Universitäten eingeworben. Ein eingeworbenes Drittmittelprojekt kann die Ausstattung wissenschaftlicher Akteur*innen mit einem Schlag erheblich steigern, und zwar in einem Ausmaß, wie dies durch die Erhöhung der Grundmittel im Normalfall nicht möglich ist (Hüther et al. 2010: 176-178). Auf der anderen Seite sind Grundmittel nicht nur weniger zweckgebunden, sondern auch langfristiger angelegt. Sie bieten für Wissenschaftler*innen eine höhere Planbarkeit sowie eine geringere Fragilität (Ziesen und Lemm 2017: 238).

Wissenschaftliche Akteur*innen benötigen materielle Ressourcen, um Forschung betreiben zu können. Eine Steigerung dieser Ressourcen ermöglicht dementsprechend in der Regel auch eine Steigerung der Forschungsleistung, die sich in den veröffentlichten Erkenntnissen niederschlägt. Durch eine Erhöhung ihres ökonomischen Kapitals verschaffen sich wissenschaftliche Akteur*innen folglich bessere Voraussetzungen für den Reputationserwerb. Auch auf direktem 
Wege - das heißt nicht erst bei Veröffentlichung und Rezeption der finanzierten Forschungsleistungen - führt eine umfangreiche Ressourcenausstattung zu der Zuschreibung von Reputation, denn materielle Ressourcen sind ein (auch für Außenstehende erkennbarer) Indikator für wissenschaftlichen Erfolg. So bescheinigt einem bspw. die Einwerbung eines DFG-Projektes, seriöse, anspruchsvolle und relevante Forschung leisten zu können, wobei hier weniger die eingeworbenen Finanzmittel für sich, sondern vielmehr die Zertifizierung durch die Gutachter*innen anhand wissenschaftlicher Kriterien im Vordergrund stehen. Zudem erleichtert eine hohe Reputation den Zugang zu materiellen Ressourcen, sodass die Mittelausstattung im Umkehrschluss auf die Reputation der darüber verfügenden Person schließen lässt (Hüther et al. 2010: 176-178).

Indem die Sicherung und Erweiterung ökonomischen Kapitals eine zentrale Handlungsorientierung wissenschaftlicher Akteur*innen darstellt, wird ihr Handeln über die Gewährung und den Entzug der Mittel steuerbar. Wer die Bereitstellung der materiellen Ressourcen kontrolliert, erhält dementsprechend die Möglichkeit die Erkenntnisproduktion - bezüglich Inhalt, Prozess, Forschungspersonal usw. - zu beeinflussen (Gläser 2012a: 8; Hüther et al. 2010: 172). Allerdings variiert der Grad der Einflussnahme durch die Mittelgeber*innen. An dem oberen Ende der Skala stehen Auftragsforschungen, in denen nicht nur die Forschungsfrage, sondern manchmal auch das erwünschte Ergebnis zumindest implizit bereits seitens der Auftraggeber*innen vorgegeben wird. Demgegenüber gibt es Mittelzuwendungen, über deren Allokation nahezu vollständig die wissenschaftliche Gemeinschaft im peer review-Verfahren entscheidet. Hierbei verfügen Mittelempfänger*innen über mehr Gestaltungsspielraum in Bezug auf die eigene Forschung, allerdings nehmen hier fachgemeinschaftliche Relevanzkriterien und Qualitätsstandards eine lenkende Wirkung ein.

Die Frage, auf welche Art Wissenschaftler*innen ihr ökonomisches Kapital erhöhen können, bedarf einer differenzierten Betrachtung. Zunächst unterliegen die persönlichen Gehalts- und Karrierestufen von Wissenschaftler*innen einer relativ engen Begrenzung. Nach Erreichung einer W3-Professur haben Wissenschaftler*innen bspw. nur eingeschränkte Möglichkeiten im Rahmen ihrer Anstellung an einer Universität oder einem Forschungsinstitut ihre Karrierestufe, ihr Gehalt oder ihre Grundausstattung zu erhöhen. Allerdings gibt es potenzielle Einnahmequellen außerhalb der Anstellung, die zumindest das persönliche Einkommen erhöhen können, wie zum Beispiel das Verfassen von Lehrbüchern, Beratungs- oder Gutachter*innentätigkeiten (Hüther et al. 2010: 173-179). Die Ausstattungsmittel auf Lehrstuhlebene, zu denen Personalmittel und Sachmittel zählen, lassen sich zum einen durch Erhöhung der zur Verfügung stehenden Grundmittel, bspw. im Zuge von Bleibeverhandlungen, und zum anderen durch 
die Einwerbung von Drittmitteln erhöhen (ebd.: 176-178). Die Ausstattungsgewinne im Rahmen von Bleibeverhandlungen sind meist geringer als diejenigen im Rahmen der erfolgreichen Einwerbung von Drittmitteln, letztere unterliegen aber auch einer stärkeren Zweckbindung und zeitlichen Begrenzung.

Spätestens seit den 1970er Jahren hat die Bedeutung von Drittmitteln zugenommen, während die Bedeutung von Grundmitteln in der Forschung abnimmt. Ursächlich dafür ist zum einen die „massification“ des Bildungssystems, die sich in einem stetigen Anstieg der Studierendenzahlen ausdrückt (Gibbons et al. 1994: 70-80). Demgegenüber stagniert die Grundausstattung der Universitäten, sodass ein steigender Lehraufwand mit gleichbleibenden materiellen Ressourcen geleistet werden muss (Ziesen und Lemm 2017: 238). Auch wenn die Grundausstattung der universitären Fachgebiete formal jeweils zur Hälfte für Forschung und Lehre vorgesehen ist, kann daher davon ausgegangen werden, dass sich die tatsächliche Verteilung der Grundmittel ,unter der Hand“ zugunsten der Lehre und zulasten der Forschung verschoben hat (Schimank 2000; Aljets 2015: 85).

Der abnehmenden Grundausstattung im Bereich Forschung steht eine steigende Verfügbarkeit von Drittmitteln gegenüber. So wird jede zweite befristete Stelle an Hochschulen mittlerweile über Drittmittel gefördert (Ziesen und Lemm 2017: 238). Die Drittmittelquote, also das Verhältnis der Drittmittel zu den Gesamteinnahmen der Hochschulen (ohne Verwaltungseinnahmen), erhöhte sich von $19 \%$ im Jahr 2003 auf 28 \% im Jahr 2015 (DFG 2018: 24-25, 2015: 25). Berücksichtigt man, dass die Ausgaben für Lehre, Infrastruktur, Instandhaltung etc. größtenteils aus den Grundmitteln finanziert werden, so lassen sich schätzungsweise mehr als $40 \%$ des Forschungsetats an deutschen Hochschulen den Drittmitteln zuschreiben (Aljets 2015: 85-86). Ein weiterer Faktor der erhöhten Bedeutung von Drittmitteln sind die gestiegenen Kosten vieler Forschungsvorhaben. Insbesondere in den physikalisch-technischen, aber auch in einigen naturund sozialwissenschaftlichen Fächern nehmen Forschungsprojekte mit hohem Ausstattungs- und Personalbedarf zu. Diese lassen sich mit den in ihrer Höhe vergleichsweise starren Grundmitteln nicht finanzieren (ebd.: 87). Die Folge des Bedeutungszuwachses von Drittmitteln ist ein starker Anstieg des Antragsaufkommens, demgegenüber die Erhöhung der Drittmittelsummen geringer ausfällt, sodass die Bewilligungsquoten stark gesunken sind. So ist bspw. im Rahmen der Einzelförderung der DFG fachübergreifend der prozentuale Anteil bewilligter Anträge gegenüber der Gesamtzahl der Anträge von 84,5 \% im Jahr 1974 auf 34,6 \% im Jahr 2018 gesunken (DFG 2019b: 195; Aljets 2015: 90).

Die sinkenden Bewilligungsquoten zusammen mit der gestiegenen Bedeutung von Drittmitteln strukturieren den Prozess der Ressourcenallokation als „QuasiMarkt", auf dem Wissenschaftler*innen zunehmend miteinander um Drittmittel 
konkurrieren (Aljets 2015: 91; Schimank und Volkmann 2008: 387-388; Whitley et al. 2018: 110). Zwar ist auch auf Seiten der Fördermittelgeber*innen eine Diversifizierung und ein zunehmender Wettbewerb um wertvolle Erkenntnisse und hochrangige Wissenschaftler*innen zu beobachten, allerdings ist der Wettbewerbsdruck auf Seiten der Wissenschaftler*innen deutlich stärker ausgeprägt, was sich an der steigenden Selektivität der Antragsbewilligung zeigt. Sie versetzt die Drittmittelgeber*innen in die Lage, selbst strategisch innerhalb der gegebenen Akteur*innenkonstellationen agieren zu können, indem sie die Projektanträge auswählen, die ihren Zielvorstellungen am ehesten entsprechen. Die seit den 1960er Jahren wachsende Überzeugung, Forschung brauche mehr politische und wirtschaftliche Orientierung, um gesellschaftlich und wirtschaftlich verwertbare Ergebnisse zu produzieren, bestärkt die Drittmittelgeber*innen darin, eigenhändig strategische Anreize für - in ihren Augen - relevante Forschungsvorhaben zu setzen. Die steigende Selektivität wird also mit einer stärkeren Konditionalität der Förderinstrumente verbunden, in der sich der inhaltliche Gestaltungswille der Förderorganisationen manifestiert (Aljets 2015: 91-92; Schimank 2005b; Gläser et al. 2015).

Es lassen sich verschiedene Dimensionen der Konditionalität unterscheiden, die bei der Mittelvergabe - insbesondere Drittmittel - in Bezug auf die Forschung zur Anwendung kommen (vgl. Aljets 2015: 90-91; Gläser 2012a). So kann die Mittelvergabe an die Erreichung bestimmter Output-Ziele geknüpft sein (Veröffentlichungen, Veranstaltungen, Einwerbung weiterer Mittel usw.). Ebenso sind Vorgaben in Bezug auf den Inhalt der Forschung (Themen, Ziele, Methodische Ansätze u.ä.) gängig. Des Weiteren kann die Bereitstellung von Forschungsmitteln an die Ausrichtung der Forschung (Interdisziplinarität, Anwendungsbezug, Innovativität u.ä.), an die Zusammenarbeit mit bestimmten Partner*innen (Industrie, Verwaltung, Zivilgesellschaft, Anwender*innen u.ä.) sowie an die Verortung in bestimmten Regionen (ländliche Räume, Europa u.ä.) geknüpft sein. Hinzu kommen die klassischen intellektuellen Kriterien (Relevanz, wissenschaftliche Qualität usw.) sowie Auswahlkriterien, nach denen die Antragstellenden beurteilt und ausgewählt werden (Exzellenz, institutionelle Zuordnung usw.). Reputation ist folglich nicht nur ein Resultat der Allokation von ökonomischem Kapital, sondern auch eine Voraussetzung, um Zugang zu bestimmten materiellen Ressourcen erhalten zu können.

Auf Seiten der Wissenschaftler*innen hat die steigende Selektivität und Konditionalität zur Folge, dass diejenigen, die auf Drittmittel angewiesen sind, zunehmend opportun agieren müssen, dass sie also Forschungsvorhaben mit Blick auf die Erfolgschancen bei Drittmittelgeber*innen auswählen und entwickeln und dabei ihre persönlichen Einschätzungen bezüglich interessanter 
und erkenntnisversprechender Forschungsfragen und -methoden gegebenenfalls zurückstellen. Gläser (2012a: 12-14) benennt einige Strategien wissenschaftlicher Akteur*innen für den Umgang mit den sich verändernden Kriterien der Ressourcenallokation. So betreiben einige Professor*innen das "Smoothing “ der Kontextbedingungen, indem sie selbst die Verfügbarkeit von Fördermitteln durch die Initiierung oder inhaltliche Ausgestaltung von Förderprogrammen hinsichtlich ihrer präferierten Inhalte beeinflussen. Durch „Buffering “ schaffen sich Akteur*innen Reserven von materiellen Ressourcen, sodass sie weniger auf inhaltlich zweckgebundene Mittel angewiesen sind. Die Antizipierung und Anpassung an die wandelnden Kontextbedingungen ist die wichtigste Strategie. So entwickeln Akteur*innen „Forschungs-Portfolios“, indem sie mehrere Forschungspfade simultan verfolgen oder potenzielle Forschungspfade entwickeln, die bei Bedarf aufgenommen werden könnten. Akteur*innen, die mehr als ein Forschungsthema in ihrem Forschungs-Portfolio haben, können nichtförderfähige Forschung einstellen und den Schwerpunkt auf förderfähige Inhalte verlegen. Ebenso können Akteur*innen ihre Forschungspfade umgestalten oder anders kontextualisieren, damit sie externe Erwartungen erfüllen. Greifen diese Strategien nicht, ,rationiert" der*die Akteur*in die Forschung, indem er*sie sich bspw. auf andere Aufgaben (Lehre oder Administration) konzentriert, Studienprojekte für Forschungszwecke nutzt oder den empirischen Aufwand reduziert (Gläser 2012a: 12-14).

Der zunehmende Drittmittelanteil in der Forschungsfinanzierung zusammen mit der gestiegenen Selektivität in der Mittelvergabe bedingt stärkere Interdependenzen zwischen dem Wissenschaftssystem und den außerwissenschaftlichen Fördermittelgeber*innen. Gemäß Heidler (2010: 15) kommen daher zunehmend Fragen in den Blick, die thematisieren, inwiefern sich die beschriebenen Konstellationen auf die Inhalte der Wissensproduktion auswirken, inwiefern die Erhöhung des Anteils der Drittmittelfinanzierung zu einer Verengung der Forschung auf den Mainstream führt und inwieweit diese einer Anhebung der wissenschaftlichen Qualität zugutekommt. Allerdings wird auch über die Bereitstellung der Grundmittel das Handeln wissenschaftlicher Akteur*innen zunehmend beeinflusst. Zwischen Bereitsteller*in und Empfänger*in (bspw. Ministerium gegenüber Fakultät, Fakultät gegenüber Lehrstuhlinhaber*in) ergibt sich ein hierarchisches Verhältnis, in dem der*die Unterhaltsträger*in Entscheidungen über den Umfang der Mittel durchsetzen und diese an Bedingungen oder Erwartungen knüpfen kann (Gläser 2012a: 9). Gemäß Whitley et al. (2018: 110) nimmt generell die Einflussnahme auf den Inhalt der Forschung zu: 
„While these preferences [towards particular priority areas and research problems] have sometimes been determined and justified in terms of their intellectual promise and significance, they increasingly are linked to public policy priorities as formulated by governments. “

\subsubsection{Die Akkumulation der wissenschaftlichen Kapitalsorten als Zyklus}

In den drei vorangegangenen Teilkapiteln wurden die drei wesentlichen Einflussgrößen des institutionellen Kontextes der Wissenschaft erläutert: Formale Koordination, Allokation von Reputation und Allokation von Ressourcen. Vermittelt über diese Kanäle lässt sich das Handeln von wissenschaftlichen Akteur*innen beeinflussen.

Analog zu den genannten Einflussgrößen lassen sich drei grundlegende Kapitalsorten unterscheiden, deren Erwerb wissenschaftliche Akteur*innen anstreben: Reputation, materielle Ressourcen (bzw. ökonomisches Kapital) und Einfluss (bzw. das formale Kapital einschließlich der Fähigkeit dieses zu nutzen). In diesen Kapitalsorten sind auch die wesentlichen Handlungsorientierungen wissenschaftlicher Akteur*innen zu sehen. Sie streben also nicht allein nach Wahrheit, relevanten Erkenntnissen oder (deren Übersetzung in) Reputation, sondern mit unterschiedlichen Gewichtungen - auch nach ökonomischem und formalem Kapital.

Es ist bereits an mehreren Stellen herausgestellt worden, dass sich die verschiedenen Kapitalsorten jeweils für bestimmte Zwecke investieren und gegeneinander eintauschen, verleihen bzw. übersetzen lassen. Wer sich bspw. ein hohes $\mathrm{Ma} ß$ an Reputation erarbeitet hat, der*die hat gute Voraussetzungen eine hochrangige Position zu erlangen oder Fördermittel einzuwerben. Eine hochrangige Position verleiht einem*einer Akteur*in wiederum Reputation, Einfluss sowie leichteren Zugang zu materiellen Ressourcen und persönlichen Bekanntschaften. Diese Faktoren lassen sich wiederum für die Produktion neuer Erkenntnisse einsetzen, was die erneute Steigerung der Reputation ermöglicht. Bourdieu (1988: 151) resümiert zu diesem Mechanismus: „Wer (Kapital) hat, der bekommt (Kapital)“. Im Rahmen seiner Kulturtheorie erkennt er in der Investition bzw. dem Austausch der Kapitalsorten eine grundlegende Strategie wissenschaftlicher Akteur*innen mit dem finalen Ziel der Maximierung ,wissenschaftlicher Autorität“, also „prestige, recognition, fame, etc. “ (Bourdieu 1975: 21; Latour und Woolgar 1982: 39-40). An dieser Stelle sei allerdings klargestellt, dass die 
Umwandlung der verschiedenen Kapitalsorten kein linearer und planbarer Prozess ist, wie die vorangegangenen Abschnitte gezeigt haben.

Eine in der Wissenschaftssoziologie weit verbreitete Theorie dieses Mechanismus der iterierenden Übersetzung verschiedener Kapitalsorten ist der „credit cycle“ (auch „cycle of credibility“, im deutschen als „Glaubwürdigkeitszyklus “ übersetzt) von Latour und Woolgar $(1982,1986)$. Unter Glaubwürdigkeit wird dabei eine Form des Kredits (,credit") verstanden, den wissenschaftliche Akteur*innen einsetzen müssen, um Wissenschaft betreiben zu können (Hessels et al. 2019: 129-130). Sie setzen diesen in jenen Forschungsbereichen ein, in denen sie den höchsten Gewinn erwarten (Debackere et al. 1994: 32-33). Akteur*innen suchen den Kreditzyklus ständig zu beschleunigen und zu erweitern, indem sie durch das Produzieren und Veröffentlichen von Erkenntnissen Reputation erwerben, diese einsetzen, um Mittel zu akquirieren, die sie investieren, um neues Wissen zu produzieren, das wiederum zu neuer Reputation führt usw. (siehe Abb. 4.1).

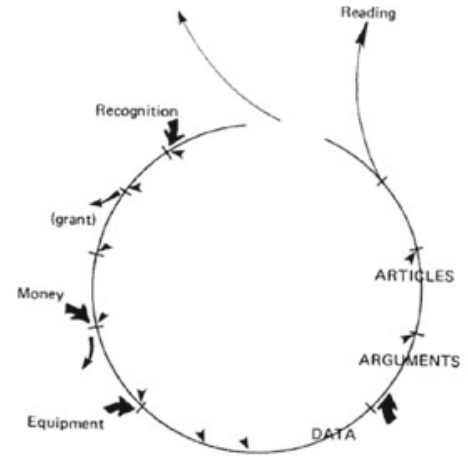

Ursprüngliche Zeichnung

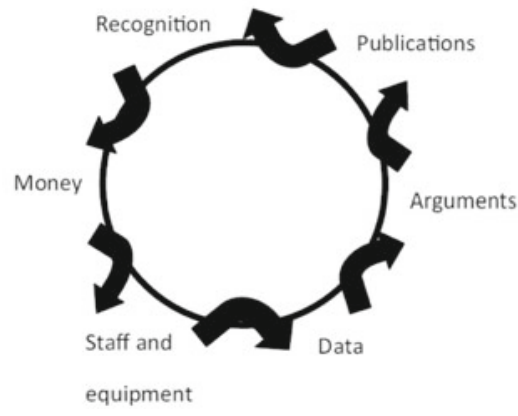

Reduzierte Darstellung

Abb.4.1 Credit cycle wissenschaftlicher Akteur*innen nach Latour und Woolgar (links: Latour und Woolgar (1982: 35), rechts: Hessels et al. (2019: 129))

Für die theoretische Einordnung des credit cycle ist es gemäß Weingart (2003: 49) wichtig zu betonen, dass es sich um keine rein machttheoretische Konzipierung der wissenschaftlichen Institutionalisierungsprozesse handelt: 


\begin{abstract}
„Eine Akkumulation nur materiellen Kapitals verschafft keine Glaubwürdigkeit in der wissenschaftlichen Kommunikation. Materielles Kapital muss erfolgreich in symbolisches (hier: innovative Forschungsergebnisse) übersetzt werden, wenn Reputation dabei entstehen soll, die wiederum die Basis für weitere Kommunikationen und die Aufmerksamkeit der Kollegen bildet. Glaubwürdigkeit lässt sich nicht durch Institutionalisierungsstrategien allein herstellen. Sie ist vielmehr das Ergebnis eines Kommunikationsprozesses, an dem immer eine Vielzahl von Wissenschaftlern teilnehmen, deren Zustimmung sich nicht erzwingen lässt. “(Weingart 2003: 49)
\end{abstract}

Dennoch wird die Theorie Latours und Woolgars dafür kritisiert, sie würde wissenschaftliche Akteur*innen auf Homines oeconomici reduzieren, die an keinem anderen Wert interessiert seien als an der Quasi-Währung credibility (Fochler 2016: 928). Weiterhin wendet bspw. Knorr-Cetina (1981: 70-74) ein, die Theorie würde sich kaum auf tagtägliche Forschungsentscheidungen (bspw. im Labor) im Sinne eines bewussten Abwägens mit dem Ziel der Maximierung der Glaubwürdigkeit anwenden lassen. Außerdem würden Mechanismen der Ausbeutung, der formalen Koordination sowie der Einfluss wissenschaftsexterner Akteur*innen ausgeblendet (Hessels et al. 2019: 130).

Diese Kritik aufgreifend, verknüpft Fochler (2016) den credit cycle mit aktuellen Theorien zum akademischen Kapitalismus sowie zum wachsenden Einfluss von New Public Management-Ansätzen in der Wissenschaft (bspw. Berman 2013; Hofmann und Hirschauer 2012). Demzufolge haben die Transformationen der letzten Jahre zugunsten einer stärker wettbewerbsorientierten Governance der Wissenschaft wissenschaftliche Akteur*innen zu Unternehmer*innen gemacht, bzw. genauer ",not necessarily entrepreneurs interested in commercializing their research, but entrepreneurial managers of their own careers, publications, and grant portfolios “ (Fochler 2016: 924). Fochler führt den Begriff „epistemic capitalism " ein, um die Mechanismen der wissenschaftlichen Kapitalakkumulation zu beschreiben, wobei Kapital nicht nur monetäre Werte umfasst, sondern grob als "forms of worth that are durable enough to deploy as capital" umschrieben wird (Rushforth et al. 2019: 211). Nicht alle Werte, die wissenschaftliche Akteur*innen als wichtig oder motivierend im Rahmen ihrer wissenschaftlichen Tätigkeit ansehen, können demnach vermarktet, akkumuliert und im Glaubwürdigkeitszyklus reinvestiert werden. Forschung, die nicht mit den aktuell vorherrschenden Wahrheitsvorstellungen und Relevanzkriterien der Fachgemeinschaft in Einklang zu bringen ist, mag zwar einen epistemischen Wert besitzen, mit dem sich einzelne Forscher*innen identifizieren können, dieser lässt sich aber nur schwerlich in Kapital transformieren (Rushforth et al. 2019: 212). Die daran anschließende Frage, inwiefern spezifische epistemische Werte und Inhalte, die sich angesichts aktueller institutioneller Anreizsysteme nicht (schnell genug) kapitalisieren und 
reinvestieren lassen, unterdrückt bzw. am Leben gehalten werden, ist dabei grundlegend für eine Reihe von Arbeiten zu den epistemischen Effekten institutioneller Anreizsysteme (für einen Überblick siehe Müller und Rijcke 2017). Fochler (2016: 943) schätzt die Entwicklungen zugunsten eines stärkeren Wettbewerbs im Zuge der New Public Management-Reformen primär als schädlich für die Wissenschaft ein:

„As a result, long-term epistemic agendas may be sacrificed to the needs of short-term productivity, true cooperation that goes beyond the exchange of authorship tokens might be discouraged, and the social fabric of scientific institutions might be ruptured by the inherent conflict between individual and collective forms of accumulation."

Die jüngeren Anwendungsbeispiele des credit cycle zeigen die Eignung des Konzepts für die Identifizierung von Mechanismen wissenschaftlicher Wissensproduktion. Hessels et al. (2019) demonstrieren dies anhand einer vergleichenden Untersuchung von vier Forschungsgruppen aus vier verschiedenen Disziplinen. Sie konzeptualisieren die unterschiedlichen Elemente des Zyklus sowie die spezifischen Eintauschmechanismen in einer aktualisierten Version des creditcycle und stellen auf Basis der unterschiedlichen Gewichtungen und Ausformungen dieser Elemente einen Vergleich der vier ,epistemic cultures “ (Knorr-Cetina 1999) an.

Über alle in Kapitel 4 genannten Ansätze hinweg lässt sich weitgehende Einigkeit bezüglich der grundlegenden Mechanismen der Wissenschaft feststellen. Unterschiede sind primär in den unterschiedlichen Perspektiven und Forschungstraditionen zu sehen und in den dementsprechend variierenden Schwerpunktsetzungen, Systematisierungen und Nomenklaturen. Es ist (mittlerweile) unbestritten, dass die wissenschaftliche Erkenntnisproduktion eine gewichtige „,unwissenschaftliche“ (,politische“, ,,strategische“, ,kapitalistische“, ,transepistemische" etc.) Komponente hat. Das heißt, es ist nicht (nur) das Streben nach neuen, wahren Erkenntnissen, das die Forscher*innen antreibt und das die Wissenschaft strukturiert, sondern (auch) die Vermehrung von Einfluss, Reputation und materiellen Ressourcen. Uneinigkeit herrscht bezüglich der Handhabung „,wissenschaftlicher" (,epistemischer", „kognitiver“, ,wissenschaftlicher", ,intellektueller", „nicht-sozialer") Prozesse und Strukturen (vgl. Gläser und Laudel 2015b). Sie werden zwar übergreifend als notwendiges Mittel zur Erlangung von Reputation, Einfluss und materiellen Ressourcen angesehen. Ob sie aber analytisch überhaupt als solche gerahmt werden, und wenn ja, ob sie auf ein untergeordnetes ,Mittel zum Zweck' reduziert werden oder gleichwertig als eine von zwei Seiten der Wissenschaft - einer epistemischen und einer strategischen - betrachtet werden, variiert von Autor*in zu Autor*in. 
Open Access Dieses Kapitel wird unter der Creative Commons Namensnennung 4.0 International Lizenz (http://creativecommons.org/licenses/by/4.0/deed.de) veröffentlicht, welche die Nutzung, Vervielfältigung, Bearbeitung, Verbreitung und Wiedergabe in jeglichem Medium und Format erlaubt, sofern Sie den/die ursprünglichen Autor(en) und die Quelle ordnungsgemäß nennen, einen Link zur Creative Commons Lizenz beifügen und angeben, ob Änderungen vorgenommen wurden.

Die in diesem Kapitel enthaltenen Bilder und sonstiges Drittmaterial unterliegen ebenfalls der genannten Creative Commons Lizenz, sofern sich aus der Abbildungslegende nichts anderes ergibt. Sofern das betreffende Material nicht unter der genannten Creative Commons Lizenz steht und die betreffende Handlung nicht nach gesetzlichen Vorschriften erlaubt ist, ist für die oben aufgeführten Weiterverwendungen des Materials die Einwilligung des jeweiligen Rechteinhabers einzuholen. 